\title{
POINTWISE CONVERGENCE TO INITIAL DATA OF HEAT AND LAPLACE EQUATIONS
}

\author{
GUSTAVO GARRIGÓS, SILVIA HARTZSTEIN, TERESA SIGNES, JOSÉ LUIS TORREA, \\ AND BEATRIZ VIVIANI
}

\begin{abstract}
Let $L$ be either the Hermite or the Ornstein-Uhlenbeck operator on $\mathbb{R}^{d}$. We find optimal integrability conditions on a function $f$ for the existence of its heat and Poisson integrals, $e^{-t L} f(x)$ and $e^{-t \sqrt{L}} f(x)$, solutions respectively of $U_{t}=-L U$ and $U_{t t}=L U$ on $\mathbb{R}_{+}^{d+1}$ with initial datum $f$. As a consequence we identify the most general class of weights $v(x)$ for which such solutions converge a.e. to $f$ for all $f \in L^{p}(v)$, and each $p \in[1, \infty)$. Moreover, if $1<p<\infty$ we additionally show that for such weights the associated local maximal operators are strongly bounded from $L^{p}(v) \rightarrow L^{p}(u)$ for some other weight $u(x)$.
\end{abstract}

\section{INTRODUCTION}

In this paper we consider the heat and Poisson semigroups, $e^{-t L}$ and $e^{-t \sqrt{L}}$, associated with the following differential operators in $\mathbb{R}^{d}$ :

$$
L \in\left\{-\Delta,-\Delta+R,-\Delta+|x|^{2}\right\}
$$

(with $R>0$ ) or with the Ornstein-Uhlenbeck operator $\mathcal{O}=-\Delta+2 x \cdot \nabla$. We consider the related partial differential equations

$$
\left\{\begin{array}{l}
u_{t}=-L u, \quad(t, x) \in(0, \mathrm{~T}) \times \mathbb{R}^{d}, \\
u(0)=f
\end{array}\right.
$$

and

$$
\left\{\begin{array}{l}
u_{t t}=L u, \quad(t, x) \in \mathbb{R}_{+}^{d+1} \\
u(0)=f,
\end{array}\right.
$$

where in the first case we allow $0<\mathrm{T} \leq \infty$.

We want to find minimal conditions on the initial datum $f$ such that

(i) $u(t, x)=e^{-t L} f(x)$ exists $\forall t \in(0, \mathrm{~T})$ and $x \in \mathbb{R}^{d}$ (as an absolutely convergent integral), and satisfies $(\mathrm{H})$,

(ii) $\lim _{t \rightarrow 0} u(t, x)=f(x)$, for a.e. $x \in \mathbb{R}^{d}$.

Received by the editors November 18, 2013 and, in revised form, August 15, 2014 and August 29, 2014.

2010 Mathematics Subject Classification. Primary 42C10, 35C15, 33C45, 40A10.

Key words and phrases. Hermite operator, Ornstein-Uhlenbeck, Poisson integral, weighted inequalities.

The first author was partially supported by grants MTM2010-16518, MTM2013-40945-P and MTM2014-57838-C2-1-P (Spain). The third author was partially supported by grants MTM201342220-P and Fundación Séneca 19378/PI/14 (Murcia, Spain). The fourth author was partially supported by Grant MTM2011-28149-C02-01 (Spain). The second and fifth authors were partially supported by grants from Consejo Nacional de Investigaciones Científicas y Técnicas (CONICET) and Universidad Nacional del Litoral (Argentina). 
The same is asked when $u(t, x)=e^{-t \sqrt{L}} f(x)$ with respect to $(\mathrm{P})$.

It is well known that these properties hold when $f \in L^{p}\left(\mathbb{R}^{d}\right)$, and more generally, when $f \in L^{p}(w)$ and $w \in A_{p}\left(\mathbb{R}^{d}\right), 1 \leq p<\infty$. Indeed, these are classically obtained from the $L^{p}(w)$-boundedness of the corresponding maximal operators

$$
h^{*} f(x)=\sup _{t>0}\left|e^{-t L} f(x)\right|, \quad P^{*} f(x)=\sup _{t>0}\left|e^{-t \sqrt{L}} f(x)\right|
$$

(see e.g. [19]). However, one expects that (i) should hold for more general functions (growing at infinity below a certain critical order), while (ii) should only be related with the boundedness of the local maximal operators

$$
h_{a}^{*} f(x)=\sup _{0<t<a}\left|e^{-t L} f(x)\right|, \quad P_{a}^{*} f(x)=\sup _{0<t<a}\left|e^{-t \sqrt{L}} f(x)\right|,
$$

for a sufficiently small $a>0$.

The goals of this paper are the following:

(I) Find the most general conditions in a function $f$ such that properties (i) + (ii) hold.

(II) Find the most general conditions in a weight $v$ such that (i) $+(i i)$ hold for all $f \in L^{p}(v)$.

(III) Show that, for all weights $v$ as in (II), the local maximal operators $h_{a}^{*}$ and $P_{a}^{*}$ map $L^{p}(v) \rightarrow L^{p}(u)$ for some other weight $u$.

When $L=-\Delta$ these questions have recently been investigated by three of the authors in [7, although parts of it can be traced back to the classical literature [20 22. For example, for the heat equation, (i) and (ii) hold for a function $f$ if and only if

$$
\int_{\mathbb{R}^{d}}|f(y)| e^{-\frac{|y|^{2}}{4 t}} d y<\infty, \quad \forall t \in(0, \mathrm{~T})
$$

In the Poisson equation the necessary and sufficient condition becomes

$$
\int_{\mathbb{R}^{d}} \frac{|f(y)|}{(1+|y|)^{d+1}} d y<\infty
$$

and in this case $(\mathrm{P})$ holds in the whole range of $t \in(0, \infty)$. These properties are elementary and can actually be proved by direct methods without resorting to maximal operators. Then, by a duality argument, the weights $v$ in question (II) are characterized by

$$
\left\|v(y)^{-\frac{1}{p}} e^{-\frac{|y|^{2}}{4 t}}\right\|_{p^{\prime}}<\infty, \forall t \in(0, \mathrm{~T}), \quad \text { or } \quad\left\|\frac{v(y)^{-\frac{1}{p}}}{(1+|y|)^{d+1}}\right\|_{p^{\prime}}<\infty .
$$

Question (III), however, is more elaborate. It is known that, from abstract Nikishin theory, the a.e.-existence of pointwise limits implies the weak boundedness of $h_{a}^{*}$ and $P_{a}^{*}$ from $L^{p}(v)$ into $L^{p, \infty}(u)$ for some weight $u=u^{(a)}$ (see [11] or [6. Ch. VI]). The strong boundedness requires deeper arguments and is related to a version of the two-weight problem studied by Carleson-Jones 5 and Rubio de Francia 13 in the 80s. Thus, a main contribution of [7] was precisely to show that (III) holds for the class of weights in (1.4), making use of the vector-valued approach developed by Rubio de Francia [13].

In this paper we shall investigate similar questions for the differential operators $L \in\left\{-\Delta+R,-\Delta+|x|^{2},-\Delta+2 x \cdot \nabla\right\}$. All of them have explicit heat kernels, from which we derive the necessary integrability conditions one must require on $f$. 
The results are summarized in Table 1, with a precise statement in the theorem below.

TABLE 1 . Necessary and sufficient conditions on $f$ for the existence of $e^{-t L} f$ and $e^{-t \sqrt{L}} f$.

\begin{tabular}{|c|c|c|}
\hline$L$ & heat & $\begin{array}{l}\text { Poisson } \\
\end{array}$ \\
\hline$-\Delta+R$ & $\begin{array}{r}\int_{\mathbb{R}^{d}}|f(y)| e^{-\frac{|y|^{2}}{4 s}} d y<\infty \\
\forall s \in(0, \mathrm{~T})\end{array}$ & $\int_{\mathbb{R}^{d}}|f(y)| \frac{e^{-\sqrt{R} \sqrt{1+|y|^{2}}}}{(1+|y|)^{\frac{d}{2}+1}} d y<\infty$ \\
\hline$-\Delta+|x|^{2}$ & $\begin{array}{r}\int_{\mathbb{R}^{d}}|f(y)| e^{-\frac{|y|^{2}}{4 s}} d y<\infty \\
\forall s \in(0, \operatorname{th}(2 \mathrm{~T}) / 2)\end{array}$ & $\int_{\mathbb{R}^{d}}|f(y)| \frac{e^{-|y|^{2} / 2}}{(1+|y|)^{\frac{d}{2}}[\ln (e+|y|)]^{3 / 2}} d y<\infty$ \\
\hline$-\Delta+2 x \cdot \nabla$ & $\begin{array}{r}\int_{\mathbb{R}^{d}}|f(y)| e^{-\left(\frac{1}{s}+2\right) \frac{|y|^{2}}{4}} d y<\infty \\
\forall s \in(0, \operatorname{th}(2 \mathrm{~T}) / 2)\end{array}$ & $\int_{\mathbb{R}^{d}}|f(y)| \frac{e^{-|y|^{2}}}{[\ln (e+|y|)]^{1 / 2}} d y<\infty$ \\
\hline
\end{tabular}

Theorem 1.1. Let $0<\mathrm{T} \leq \infty$ (and $\mathrm{T}=\infty$ in the Poisson case). Let $f: \mathbb{R}^{d} \rightarrow \mathbb{C}$ be a measurable function such that one of the conditions in Table 1 holds. Then, the corresponding heat or Poisson integral, denoted $u(t, x)$, defines an absolutely convergent integral such that

(i) $u \in C^{\infty}\left((0, \mathrm{~T}) \times \mathbb{R}^{d}\right)$ and satisfies the corresponding pde $(\mathrm{H})$ or $(\mathrm{P})$,

(ii) $\lim _{t \rightarrow 0^{+}} u(t, x)=f(x)$, for a.e. $x \in \mathbb{R}^{d}$.

Conversely, if a function $f \geq 0$ is such that one of the following holds:

- $e^{-t L} f\left(x_{t}\right)<\infty$, for each $t \in(0, \mathrm{~T})$ and some $x_{t} \in \mathbb{R}^{d}$, or

- $e^{-t \sqrt{L}} f(x)<\infty$ for some $(t, x) \in(0, \infty) \times \mathbb{R}^{d}$,

then $f$ must necessarily satisfy the corresponding condition in Table 1 .

For completeness we have stated the results for both the heat and Poisson equations, although in the heat setting some of the results are already known; see [1. Our main contribution concerns the Poisson equation. The main issue here is that the Poisson kernel is not so explicit, but defined via a subordination formula

$$
e^{-t \sqrt{L}}=\frac{t}{\sqrt{4 \pi}} \int_{0}^{\infty} e^{-\frac{t^{2}}{4 u}} e^{-u L} \frac{d u}{u^{3 / 2}}, \quad t>0
$$

(see e.g. [15, p. 46]). The characterization will be obtained from very precise estimates on this kernel, which seem new in the literature and we think are of independent interest (see $\$ 4.1$ below). We refer to recent work of Liu and Sjögren [10] for different and more general bounds of the Poisson kernel related with the Ornstein-Uhlenbeck operator $\mathcal{O}$.

We include a few comments about Theorem 1.1. First, the conditions required on $f$ for the existence of Poisson integrals are always stronger than for heat integrals, in fact strong enough to guarantee the existence of the latter in the whole upper-half plane $(0, \infty) \times \mathbb{R}^{d}$ (as is perhaps expected from the subordination formula (1.5)). Also, unlike the classical case in (1.3), exponential growth of $f$ is allowed in Poisson integrals, as it is already apparent when $L=-\Delta+R$, with $R>0$.

\footnotetext{
${ }^{1}$ Observe that the condition for $L=-\Delta+R$ in Table 1 can equivalently be written as $\int_{\mathbb{R}^{d}}|f(y)|(1+\sqrt{R}|y|)^{\frac{d}{2}}(1+|y|)^{-(d+1)} e^{-\sqrt{R} \sqrt{1+|y|^{2}}} d y<\infty$, which for $R=0$ recovers (1.3).
} 
As illustrative examples, in the Hermite case $L=-\Delta+|x|^{2}$, a Gaussian initial datum $f(y)=e^{|y|^{2} / 2}$ is admissible for the existence of $e^{-t L} f$ for all $t>0$, while the existence of $e^{-t \sqrt{L}} f$ requires a slightly slower growth, such as $f(y)=e^{|y|^{2} / 2} /$ $(1+|y|)^{d / 2}$. Similarly, in the Ornstein-Uhlenbeck setting, $e^{-t \mathcal{O}} f$ is well defined for all $t>0$ when $f(y)=(1+|y|)^{N} e^{|y|^{2}}$ and $N \in \mathbb{N}$, even if these functions are not in $L^{1}(d \gamma)$. Thus we can cover more functions than the classes considered in [8, §3]. The same applies to the Poisson integrals, since $e^{-t \sqrt{\mathcal{O}}} f$ exists when $f(y)=e^{|y|^{2}} /\left[(1+|y|)^{d} \ln (e+|y|)\right]$, which is not in $L^{1}(d \gamma)$.

We now turn to the questions involving weighted spaces $L^{p}(v)$. We wish to describe the classes

$$
D_{p}^{\text {heat }}(L) \quad \text { and } \quad D_{p}^{\text {Pois }}(L)
$$

of all weights $v: \mathbb{R}^{d} \rightarrow(0, \infty)$ such that the corresponding properties (i) +(ii) hold for all functions $f \in L^{p}(v)$. These are easily characterized from Theorem 1.1 . Indeed, suppose we want to meet a condition in Table 1 written in the form

$$
\int_{\mathbb{R}^{d}}|f(y)| \varphi(y) d y<\infty
$$

for a suitable $\varphi$ and all $f \in L^{p}(v)$. Then, a sufficient condition on the weight $v$ is

$$
v^{-\frac{1}{p}} \varphi \in L^{p^{\prime}}\left(\mathbb{R}^{d}\right) \text {. }
$$

That this condition is also necessary follows by a simple duality argument (see [4, p. 10]). To write this in a unified way, we give precise definitions of $\varphi$ in Table 2 .

TABLE 2. Integrability factors $\varphi_{s}$ and $\varphi$, for each operator $L$.

\begin{tabular}{|c|c|c|}
\hline$L$ & heat & Poisson \\
\hline$-\Delta+R$ & $\varphi_{s}(y)=e^{-\frac{|y|^{2}}{4 s}}, 0<s<\mathrm{T}^{*}=\mathrm{T}$ & $\varphi(y)=\frac{e^{-\sqrt{R} \sqrt{1+|y|^{2}}}}{(1+|y|)^{\frac{d}{2}+1}}$ \\
\hline$-\Delta+|x|^{2}$ & $\varphi_{s}(y)=e^{-\frac{|y|^{2}}{4 s}}, 0<s<\mathrm{T}^{*}=\frac{\operatorname{th} 2 \mathrm{~T}}{2}$ & $\varphi(y)=\frac{e^{-|y|^{2} / 2}}{(1+|y|)^{\frac{d}{2}}[\ln (e+|y|)]^{3 / 2}}$ \\
\hline$-\Delta+2 x \cdot \nabla$ & $\varphi_{s}(y)=e^{-\frac{|y|^{2}}{4}\left(\frac{1}{s}+2\right)}, 0<s<\mathrm{T}^{*}=\frac{\operatorname{th} 2 \mathrm{~T}}{2}$ & $\varphi(y)=\frac{e^{-|y|^{2}}}{[\ln (e+|y|)]^{1 / 2}}$ \\
\hline
\end{tabular}

Corollary 1.2. Let $1 \leq p<\infty$. Then, with the notation in Table 2,

$$
v \in D_{p}^{\text {heat }}(L) \quad \Leftrightarrow \quad\left\|v^{-\frac{1}{p}} \varphi_{s}\right\|_{L^{p^{\prime}\left(\mathbb{R}^{d}\right)}}<\infty, \quad \forall s \in\left(0, \mathrm{~T}^{*}\right),
$$

and

$$
v \in D_{p}^{\text {Pois }}(L) \quad \Leftrightarrow \quad\left\|v^{-\frac{1}{p}} \varphi\right\|_{L^{p^{\prime}\left(\mathbb{R}^{d}\right)}}<\infty .
$$

Our next result concerns the strong boundedness from $L^{p}(v)$ to some $L^{p}(u)$ of the local maximal operators defined in (1.2).

\footnotetext{
${ }^{2}$ Here $d \gamma(y)=e^{-|y|^{2}} d y$ is the Gaussian measure.
} 
Theorem 1.3. Let $0<\mathrm{T} \leq \infty$ (or $\mathrm{T}=\infty$ in the Poisson case) and $1<p<\infty$.

- If $v \in D_{p}^{\text {heat }}(L)$, then, for every $a \in(0, \mathrm{~T})$, there exists a weight $u=u^{(a)}$ such that

$$
h_{a}^{*}: L^{p}(v) \rightarrow L^{p}(u) \quad \text { boundedly }
$$

Moreover, there exists $\sigma_{0}=\sigma_{0}\left(a, \mathrm{~T}^{*}\right) \in(0,1)$ such that, for any $\sigma<\sigma_{0}$, the weight $u$ can be chosen such that, in addition, $u^{\sigma} \in D_{p}^{\text {heat }}(L)$.

Conversely, if (1.8) holds for some weight $u^{(a)}$ and each $a \in(0, \mathrm{~T})$, then $v \in$ $D_{p}^{\text {heat }}(L)$.

- If $v \in D_{p}^{\mathrm{Pois}}(L)$, then there exists a weight $u$ such that, for every $a>0$,

$$
P_{a}^{*}: L^{p}(v) \rightarrow L^{p}(u) \text { boundedly. }
$$

If $\sigma<1$ we can find $u$ such that, in addition, $u^{\sigma} \in D_{p}^{\text {Pois }}(L)$.

Conversely, if (1.9) holds for some $a>0$ and some weight $u$, then $v \in D_{p}^{\text {Pois }}(L)$.

As in the classical case, proving, say, (1.9) is much harder than proving the weak boundedness of $P_{a}^{*}: L^{p}(v) \rightarrow L^{p, \infty}(U)$ for some weight $U$ (although both turn out to be equivalent). For the latter, if $v \in D_{p}^{\text {Pois }}(L)$, then the existence of pointwise limits in (ii) for all $f \in L^{p}(v)$ implies that

$$
P_{a}^{*} f(x)<\infty, \quad \text { a.e. } x \in \mathbb{R}^{d}, \quad \forall f \in L^{p}(v) .
$$

Then, the assertion follows from Nikishin's theorem as stated in 6, Corollary VI.2.7]. The existence of a weight $u$ guaranteing strong convergence is more difficult and requires the use of the vector-valued machinery of Rubio de Francia [13. In fact, we need to prove new local versions of his results, which are stated separately in $₫ 2$. We finally remark that although this method gives no explicit expression for the weight $u$, we are able to show that it is "almost" in the same $D_{p}$ class as $v$; namely, for every $\varepsilon>0$ we can choose a weight $u$ such that $u^{1-\varepsilon} \in D_{p}$ (this is always the case in the Poisson setting and also in the heat setting if $a$ is sufficiently small or $\mathrm{T}^{*}=\infty$; see Remark 3.6). This result is new, even in the special cases already considered in the literature [1,7].

The paper is structured as follows. In $\oint 2$ we extend the two-weight theorem of Rubio de Francia to local maximal functions. In $\$ 3$ we prove in detail Theorems 1.1 and 1.3 for the heat equation associated with $L=-\Delta+|x|^{2}$. In 84 we do the same for the Poisson equation. In $\S \$ 5$ and 6 , respectively, we prove Theorems 1.1 and 1.3 for the operators $-\Delta+R$ and $\mathcal{O}$. Finally, in $\S 7$ we state some further remarks. Throughout the paper $A \lesssim B$ means that $A \leq c B$ for some constant $c$, which may depend on fixed parameters (such as $d, p, a$ or $R$ ), but not on the variables $t, x, y$. Dependence on the variables is stressed by the notation $C_{x}, C_{t, x}$, etc. Finally, $A \approx B$ will denote both $A \lesssim B$ and $B \lesssim A$.

\section{A tWo-Weight PRoblem For the local HaRdy-LitTleWood operator}

Let $R>1$, which we assume fixed throughout this section. We consider the following local Hardy-Littlewood maximal function:

$$
\mathcal{M}_{R}^{\text {loc }} f(x):=\sup _{r>0} \frac{1}{\left|B_{r}\right|} \int_{B_{r}(x)} f(y) \chi_{\{|y| \leq R \max (|x|, 1)\}}(y) d y .
$$

We shall adapt the arguments given by Rubio de Francia in [13] (see also [6, Ch. VI.6]) to prove the following. 
Theorem 2.1. If $1<p<\infty$ and $v^{-\frac{1}{p}} \in L_{\mathrm{loc}}^{p^{\prime}}\left(\mathbb{R}^{d}\right)$, then there exists a weight $u$ such that

$$
\mathcal{M}_{R}^{\text {loc }}: L^{p}(v) \rightarrow L^{p}(u) \quad \text { boundedly. }
$$

Moreover, if we assume that $\left\|v^{-\frac{1}{p}} e^{-A|y|^{2}}\right\|_{p^{\prime}}<\infty$, for all $A>A_{0}$ (and some fixed $\left.A_{0} \geq 0\right)$, then for every $\sigma<1$ and $b>1$ we can choose the weight $u$ such that, in addition,

$$
\left\|u^{-\frac{\sigma}{p}} e^{-A|y|^{2}}\right\|_{p^{\prime}}<\infty, \quad \forall A>A_{0} \sigma R^{2} b^{2} .
$$

In particular, if $A_{0}=0$ or $\sigma \leq 1 /(b R)^{2}$, then (2.3) holds for all $A>A_{0}$.

Proof. Following the strategy in [13], one first proves, for every $s<1$, a vectorvalued estimate

$$
\left\|\left(\sum_{j}\left|\mathcal{M}_{R}^{\mathrm{loc}} f_{j}\right|^{p}\right)^{\frac{1}{p}}\right\|_{L^{s}\left(E_{k}\right)} \leq C_{k}\left(\sum_{j}\left\|f_{j}\right\|_{L^{p}(v)}^{p}\right)^{\frac{1}{p}},
$$

for a suitable partition of $\mathbb{R}^{d}=\bigcup_{k=0}^{\infty} E_{k}$ and some constants $C_{k}$ (which may depend on $v$ and of course on $p, s$ and $R$ ). This inequality implies, by the factorization theorem of Rubio de Francia (see [6, Thm. VI.4.2]), the existence of some weight $U_{k}$, supported in $E_{k}$, such that $\left\|U_{k}^{-1}\right\|_{L^{\frac{s}{p-s}}} \leq 1$ and

$$
\int_{E_{k}}\left|\mathcal{M}_{R}^{\text {loc }} f\right|^{p} U_{k}(x) d x \leq C_{k}^{p}\|f\|_{L^{p}(v)}^{p} .
$$

In such case, to obtain (2.2) it suffices to consider the weight $u$ defined by

$$
u(x)=\sum_{k=0}^{\infty} \frac{1}{\left(2^{\gamma k} C_{k}\right)^{p}} U_{k}(x) \chi_{E_{k}}(x),
$$

for some $\gamma>0$. We now prove (2.4), with a precise expression for $C_{k}$ which later will lead to the bound in (2.3). Consider

$$
E_{0}=\{|x|<1\} \quad \text { and } \quad E_{k}=\left\{b^{k-1} \leq|x|<b^{k}\right\}, \quad k=1,2, \ldots,
$$

where $b>1$ is as in the statement. For fixed $k \in \mathbb{N} \cup\{0\}$, we split

$$
f=f \chi_{\left\{|y| \leq R b^{k}\right\}}+f \chi_{\left\{|y|>R b^{k}\right\}}=f^{\prime}+f^{\prime \prime} .
$$

It is clear that

$$
\mathcal{M}_{R}^{\text {loc }} f^{\prime \prime}(x)=0, \quad \forall x \in E_{k} .
$$

Next, using Kolmogorov's inequality [6, p. 485], followed by the Fefferman-Stein estimate for the standard Hardy-Littlewood maximal operator $\mathcal{M}$ [17, p. 56] gives

$$
\begin{aligned}
\left\|\left(\sum_{j}\left|\mathcal{M}_{R}^{\text {loc }} f_{j}\right|^{p}\right)^{\frac{1}{p}}\right\|_{L^{s}\left(E_{k}\right)} & \leq c_{s}\left|E_{k}\right|^{\frac{1}{s}-1}\left\|\left(\sum_{j}\left|\mathcal{M} f_{j}^{\prime}\right|^{p}\right)^{\frac{1}{p}}\right\|_{L^{1, \infty}} \\
& \leq c_{s, p}\left|E_{k}\right|^{\frac{1}{s}-1}\left\|\left(\sum_{j}\left|f_{j}^{\prime}\right|^{p}\right)^{\frac{1}{p}}\right\|_{L^{1}} \\
& \leq c_{s, p}\left|E_{k}\right|^{\frac{1}{s}-1}\left(\sum_{j}\left\|f_{j}\right\|_{L^{p}(v)}^{p}\right)^{\frac{1}{p}} V_{k} .
\end{aligned}
$$

The last step follows by Hölder's inequality if we set

$$
V_{k}=\left(\int_{|y|<R b^{k}} v^{-\frac{p^{\prime}}{p}}\right)^{\frac{1}{p^{\prime}}}
$$


(we also used that $f_{j}^{\prime}$ are supported in $\left\{|y|<R b^{k}\right\}$ ). Thus we have shown (2.4) with $C_{k}=c_{s, p}\left|E_{k}\right|^{\frac{1}{s}-1} V_{k}$, which are finite numbers since $v^{-\frac{1}{p}} \in L_{\mathrm{loc}}^{p^{\prime}}$.

We now turn to prove (2.3) under the assumption

$$
\|v\|_{D_{p, A}}:=\left\|v^{-\frac{1}{p}} e^{-A|y|^{2}}\right\|_{p^{\prime}}<\infty,
$$

for all $A>A_{0}$. For any such $A$, we can bound the constants $V_{k}$ by

$$
V_{k}=\left\|v^{-\frac{1}{p}} e^{-A|y|^{2}} e^{A|y|^{2}} \chi_{\left\{|y|<R b^{k}\right\}}\right\|_{p^{\prime}} \leq\|v\|_{D_{p, A}} e^{A\left|R b^{k}\right|^{2}} .
$$

This is relevant when we construct the weight $u$ as in (2.5), for which we have freedom to choose $s<1$ and $b>1$. Given $\sigma<1$, we first select $s<1$ such that $\frac{\sigma p^{\prime}}{p}=\frac{s}{p-s}$. Then,

$$
\begin{aligned}
\left\|u^{\sigma}\right\|_{D_{p, B}}^{p^{\prime}} & =\int_{\mathbb{R}^{d}} u(y)^{-\frac{\sigma p^{\prime}}{p}} e^{-p^{\prime} B|y|^{2}} d y \\
& =\sum_{k=0}^{\infty}\left(2^{\gamma k} C_{k}\right)^{\sigma p^{\prime}} \int_{E_{k}} U_{k}(y)^{-\frac{s}{p-s}} e^{-p^{\prime} B|y|^{2}} d y \\
& \leq c \sum_{k=0}^{\infty}\left(2^{\gamma k}\left|E_{k}\right|^{\frac{1}{s}-1} e^{A\left|R b^{k}\right|^{2}}\right)^{\sigma p^{\prime}} e^{-p^{\prime} B\left|b^{k-1}\right|^{2}}
\end{aligned}
$$

where in the last line we have used that $\left\|U_{k}^{-1}\right\|_{L^{\frac{s}{p-s}}} \leq 1$. Now, this series is convergent provided that

$$
B>b^{2} A R^{2} \sigma,
$$

and this is always the case if $B>A_{0} R^{2} \sigma b^{2}$ and we select $A$ close enough (but larger) than $A_{0}$.

Remark 2.2. The theorem continues to hold if we replace the condition in the weight by $\left\|v^{-\frac{1}{p}} e^{-A|y|}\right\|_{p^{\prime}}<\infty$, for all $A>A_{0}$. Indeed, in such case the same proof as above would give, for every $\sigma<1$, a weight $u$ such that $\left\|u^{-\frac{\sigma}{p}} e^{-A|y|}\right\|_{p^{\prime}}<\infty$, $\forall A>A_{0} R \sigma b$. This version of Theorem 2.1 (with $A_{0}=0$ ) will be used in 95 below.

Remark 2.3. Theorem 2.1 cannot be true for $p=1$, at least if one expects "reasonable" weights $u$ and $v$, say, such that $L^{1}(u) \subset L_{\text {loc }}^{1}\left(\mathbb{R}^{d}\right)$ and $v$ is essentially constant near some point $x_{0}$. Indeed, in such case the function

$$
f(x)=\left|x-x_{0}\right|^{-d}\left[\ln \left(e /\left|x-x_{0}\right|\right)\right]^{-2} \chi_{B_{\varepsilon}\left(x_{0}\right)} \in L^{1}(v)
$$

for some $\varepsilon>0$, but

$$
\mathcal{M}_{R}^{\text {loc }} f(x) \gtrsim\left|x-x_{0}\right|^{-d}\left[\ln \left(e /\left|x-x_{0}\right|\right)\right]^{-1} \text { near } x_{0},
$$

which is not locally integrable.

\section{The heat equation for the Hermite operator $L=-\Delta+|x|^{2}$}

This case was studied earlier in [1, although from a slightly different perspective. Namely, in that paper the authors seek minimal conditions on a function $f$ so that there exists some $\varepsilon>0$ for which $e^{-t L} f(x)$ is well defined up to time $\varepsilon$ and $e^{-t L} f(x) \rightarrow f(x)$ for a.e. $x$. This is satisfactory if one only cares about pointwise convergence to the initial data, but does not guarantee that the candidate solution 
$e^{-t L} f$ actually exists in a fixed band of time $t \in(0, \mathrm{~T})$. In our approach, we first fix $\mathrm{T}>0$ and the corresponding pde

$$
\left\{\begin{array}{l}
u_{t}=-L u, \quad t \in(0, \mathrm{~T}) \\
u(0)=f
\end{array}\right.
$$

and search for minimal assumptions in $f$ so that $u(t, x)=e^{-t L} f(x)$ solves the equation in the full band $t \in(0, \mathrm{~T})$, and secondly, it satisfies $e^{-t L} f(x) \rightarrow f(x)$ a.e. This more precise approach is also slightly more general than [1], since their class of admissible initial data $f$ will coincide with the union of our classes as $\mathrm{T}>0$ varies.

3.1. Kernel estimates. The kernel $h_{t}(x, y)$ of $e^{-t L}$ (usually called Mehler kernel) has a well-known explicit expression which we shall write in the form

$$
h_{t}(x, y)=\left(\frac{1-s^{2}}{4 \pi s}\right)^{\frac{d}{2}} e^{-\frac{1}{4}\left(\frac{|x-y|^{2}}{s}+s|x+y|^{2}\right)} \quad \text { with } s=\operatorname{th} t .
$$

We shall use the following estimates.

Lemma 3.1. Given $M>1, t>0$ and $x \in \mathbb{R}^{d}$, there exists some $C=C_{x, t, M}>0$ such that

$$
\frac{1}{C} e^{-\frac{|x-y|^{2}}{4}\left[\frac{1}{s}+s\left(\frac{M+1}{M-1}\right)^{2}\right]} \leq h_{t}(x, y) \leq C e^{-\frac{|x-y|^{2}}{4}\left[\frac{1}{s}+s\left(\frac{M-1}{M+1}\right)^{2}\right]}, \quad \forall y \in \mathbb{R}^{d},
$$

where we have set $s=\operatorname{th} t$.

Proof. Assume first that $|y| \geq M|x|$. Then, an elementary use of triangle inequalities gives $\frac{M-1}{M}|y| \leq|x \pm y| \leq \frac{M+1}{M}|y|$, and therefore

$$
\frac{M-1}{M+1}|x-y| \leq|x+y| \leq \frac{M+1}{M-1}|x-y| .
$$

Inserting these inequalities into the Mehler kernel (3.1) one easily obtains (3.2). If we assume $|y| \leq M|x|$, then the function $y \mapsto h_{t}(x, y) e^{\frac{|x-y|^{2}}{4}\left[\frac{1}{s}+s\left(\frac{M+1}{M-1}\right)^{2}\right]}$ is continuous and non-vanishing, and hence bounded from below in the compact set $|y| \leq M|x|$ by a constant $c(x, t, M)>0$. One argues similarly for the upper bound.

Remark 3.2. Observe that every $f \geq 0$ which has $e^{-t L} f(x)<\infty$, for some $x$ and $t>0$, is necessarily locally integrable. Indeed, if $y$ belongs to compact set $K$, then $h_{t}(x, y)$ is bounded below by some $c=c(t, x, K)>0$, and hence $\int_{K}|f| \leq$ $\frac{1}{c} \int h_{t}(x, y)|f(y)| d y<\infty$.

3.2. Proof of Theorem $\mathbf{1 . 1}$ for the Hermite heat equation. Theorem 1.1. in the setting considered in this section, is a direct consequence of the next three propositions. Our argument is more direct than that in [1] and also valid in greater generality.

Proposition 3.3. Let $\mathrm{T}>0$ be fixed (possibly $\mathrm{T}=\infty$ ). Then, the following are equivalent:

(i) $\int_{\mathbb{R}^{d}} h_{t}(x, y)|f(y)| d y<\infty$, for all $t \in(0, \mathrm{~T})$ and $x \in \mathbb{R}^{d}$;

(ii) $\int_{\mathbb{R}^{d}} h_{t}\left(x_{t}, y\right)|f(y)| d y<\infty$, for all $t \in(0, \mathrm{~T})$ and some $x_{t} \in \mathbb{R}^{d}$;

(iii) $\int_{\mathbb{R}^{d}}|f(y)| e^{-\frac{|y|^{2}}{4 s}} d y<\infty$, for all $s \in(0, \operatorname{th}(2 T) / 2)$. 
Proof. Clearly (i) implies (ii). We show that (ii) implies (iii). Pick any $t_{0}<\mathrm{T}$ and $x$ such that

$$
\int_{\mathbb{R}^{d}} h_{t_{0}}(x, y)|f(y)| d y<\infty .
$$

Call $s_{0}=$ th $t_{0}$, and take any $s<\operatorname{th}\left(2 t_{0}\right) / 2=\left(\operatorname{th} t_{0}+\frac{1}{\operatorname{th} t_{0}}\right)^{-1}$, that is, $\frac{1}{s}>s_{0}+\frac{1}{s_{0}}$. Then there must exist some large $M>1$ so that $\frac{1}{s}>\frac{1}{s_{0}}+s_{0}\left(\frac{M+1}{M-1}\right)^{2}$. The lower bound in (3.2) then gives

$$
e^{-\frac{|x-y|^{2}}{4 s}} \leq e^{-\frac{|x-y|^{2}}{4}\left[\frac{1}{s_{0}}+s_{0}\left(\frac{M+1}{M-1}\right)^{2}\right]} \leq C_{x, t_{0}, M} h_{t_{0}}(x, y) .
$$

Hence

$$
\int_{\mathbb{R}^{d}} e^{-\frac{|x-y|^{2}}{4 s}}|f(y)| d y<\infty, \quad \forall s<\operatorname{th}\left(2 t_{0}\right) / 2 .
$$

Using the similar but more elementary estimate

$$
\frac{1}{c} e^{-\frac{|y|^{2}}{4 s}\left(\frac{M+1}{M}\right)^{2}} \leq e^{-\frac{|x-y|^{2}}{4 s}} \leq c e^{-\frac{|y|^{2}}{4 s}\left(\frac{M-1}{M}\right)^{2}}, \quad y \in \mathbb{R}^{d},
$$

with some $c=c(x, s, M)>0$, one can place $x=0$ in (3.3). Since $t_{0}<\mathrm{T}$ is arbitrary, one obtains the assertion in (iii).

Next we show that (iii) implies (i). Pick $t_{0}<\mathrm{T}$ and $x \in \mathbb{R}^{d}$. Setting $s_{0}=$ th $\left(2 t_{0}\right) / 2$, we see that (iii) (suitably combined with (3.4)) implies that

$$
\int_{\mathbb{R}^{d}}|f(y)| e^{-\frac{|x-y|^{2}}{4 s_{0}}} d y<\infty .
$$

Take any $t<t_{0}$ and let $s=\operatorname{th} t<\operatorname{th} t_{0}$. Then $\frac{1}{s}+s>\frac{1}{\operatorname{th} t_{0}}+\operatorname{th} t_{0}=\frac{1}{s_{0}}$. Thus, there is some large $M$ such that $\frac{1}{s}+s\left(\frac{M-1}{M+1}\right)^{2}>\frac{1}{s_{0}}$, so the upper bound in (3.2) gives

$$
h_{t}(x, y) \leq C e^{-\frac{|x-y|^{2}}{4}\left[\frac{1}{s}+s\left(\frac{M-1}{M+1}\right)^{2}\right]} \leq C e^{-\frac{|x-y|^{2}}{4 s_{0}}} .
$$

Then (i) follows from (3.5).

Proposition 3.4. If $f$ satisfies the conditions in Proposition 3.3, then

$$
u(t, x)=\int_{\mathbb{R}^{d}} h_{t}(x, y) f(y) d y \in C^{\infty}\left((0, \mathrm{~T}) \times \mathbb{R}^{d}\right) .
$$

Proof. It suffices to prove that, for each $x$ and $t$,

$$
\int_{\mathbb{R}^{d}}\left|\partial_{t}^{L} \Delta_{x}^{M}\left[h_{t}(x, y)\right]\right||f(y)| d y<\infty, \quad \forall L, M \geq 0 .
$$

Since $h_{t}(x, y)$ satisfies the Hermite equation, we can assume that $M=0$. Since $s=$ th $t$ is a diffeomorphism, we may just prove (3.6) replacing $\partial_{t}$ by $\partial_{s}$. Now, from the explicit formula (3.1), this would be a consequence of

$$
\int_{\mathbb{R}^{d}}|x \pm y|^{2 L} h_{t}(x, y)|f(y)| d y<\infty, \quad \forall L \geq 0 .
$$

But if $f$ satisfies (i) of Proposition 3.3. so does $|x \pm y|^{2 L} f(y)$, so (3.7) is indeed true.

Proposition 3.5. If $f$ satisfies the conditions in Proposition 3.3, then

$$
\lim _{t \rightarrow 0^{+}} e^{-t L} f(x)=f(x), \quad \text { a.e. } x \in \mathbb{R}^{d} .
$$


Proof. It suffices to show (3.8) for a.e. $|x| \leq n$ and every fixed $n \in \mathbb{N}$. Split

$$
f=f \chi_{\{|y| \leq 2 n\}}+f \chi_{\{|y|>2 n\}}=f_{1}+f_{2} .
$$

We first show that $\lim _{t \rightarrow 0} e^{-t L} f_{2}(x)=0$ for all $|x| \leq n$. Indeed, if $|y|>2 n$ we must have $|x-y| \geq|y| / 2$, and therefore, from the explicit formula (3.1),

$$
h_{t}(x, y) \leq \frac{e^{-\frac{|x-y|^{2}}{4 s}}}{(4 \pi s)^{d / 2}} \leq \frac{e^{-\frac{|y|^{2}}{16 s}}}{(4 \pi s)^{d / 2}} \leq \frac{e^{-\frac{|y|^{2}}{32 s}-\frac{|y|^{2}}{\left.4\right|_{0}}}}{(4 \pi s)^{d / 2}} \leq c_{d} s^{-d / 2} e^{-\frac{n^{2}}{8 s}} e^{-\frac{|y|^{2}}{4 s_{0}}}
$$

if we assume $s=\operatorname{th} t \leq s_{0} / 8$ and say $s_{0}=\operatorname{th}(2 \mathrm{~T}) / 4$. Now, since $e^{-\frac{|y|^{2}}{4 s_{0}}} f(y) \in$ $L^{1}\left(\mathbb{R}^{d}\right)$ and $\lim _{s \rightarrow 0} s^{-d / 2} e^{-\frac{n^{2}}{8 s}}=0$, we obtain

$$
\left|e^{-t L} f_{2}(x)\right| \leq c_{d} s^{-d / 2} e^{-\frac{n^{2}}{8 s}} \int_{\mathbb{R}^{d}}|f(y)| e^{-\frac{|y|^{2}}{4 s_{0}}} d y \rightarrow 0, \quad \text { as } \quad t \rightarrow 0 .
$$

On the other hand, every $f$ verifying the conditions in Proposition 3.3 is locally integrable (by Remark $\underline{3.2}$ ), so by the standard pointwise convergence for $L^{1}$ functions we have

$$
\lim _{t \rightarrow 0} e^{-t L} f_{1}(x)=f(x), \quad \text { a.e. }|x| \leq n .
$$

3.3. Proof of Theorem $\mathbf{1 . 3}$ for the Hermite heat equation. This requires a more detailed proof, as the estimate in the weight $u$ is new. Let $1<p<\infty$ and $a<\mathrm{T}$ be fixed. For a weight $v$ such that $\left\|v^{-\frac{1}{p}} e^{-\frac{|y|^{2}}{4 s}}\right\|_{p^{\prime}}<\infty$ for all $s<\operatorname{th}(2 \mathrm{~T}) / 2$, we need to show that the maximal operator

$$
h_{a}^{*} f(x)=\sup _{0<t<a} \int_{\mathbb{R}^{d}} h_{t}(x, y)|f(y)| d y
$$

maps boundedly $L^{p}(v) \rightarrow L^{p}(u)$ for some weight $u$. Moreover, if $\sigma \leq \sigma_{0}$ (for a suitable $\sigma_{0}$ to be chosen) we must find a weight $u$ such that $\left\|u^{-\frac{\sigma}{p}} e^{-\frac{|y|^{2}}{4 s}}\right\|_{p^{\prime}}<\infty$ for all $s<\operatorname{th}(2 \mathrm{~T}) / 2$.

To do so, we fix $M>1$ (to be chosen later) and split the operator $h_{a}^{*}$ into two parts:

$$
\begin{aligned}
h_{a}^{*} f(x) \leq & \sup _{0<t<a} \int_{|y| \leq M \max \{|x|, 1\}} h_{t}(x, y)|f(y)| d y \\
& +\sup _{0<t<a} \int_{|y|>M \max \{|x|, 1\}} h_{t}(x, y)|f(y)| d y \\
= & A f(x)+B f(x) .
\end{aligned}
$$

For the operator $B$ we estimate the kernel as in (3.9), but with a slightly more precise computation. Now we assume $|y|>M \max \{|x|, 1\}$, so we have $|x \pm y| \geq$ $\frac{M-1}{M}|y|$. Thus, if $s=\operatorname{th} t$,

$$
\begin{aligned}
h_{t}(x, y) & \leq \frac{e^{-\left(\frac{M-1}{M}\right)^{2}(s+1 / s) \frac{|y|^{2}}{4}}}{(4 \pi s)^{d / 2}} \leq \frac{c_{M}}{|y|^{d}} e^{-\left(\frac{M-1}{M}\right)^{3}(s+1 / s) \frac{|y|^{2}}{4}} \\
& \leq \frac{c_{M}^{\prime}}{\max \left\{1,|x|^{d}\right\}} e^{-\left(\frac{M-1}{M}\right)^{3}(s+1 / s) \frac{|y|^{2}}{4}} .
\end{aligned}
$$


We pick $M$ large enough so that $\left(\frac{M}{M-1}\right)^{3}(s+1 / s)^{-1} \leq s_{0}:=\left(\frac{M}{M-1}\right)^{3} \frac{\text { th }(2 a)}{2}<\mathrm{T}^{*}=$ th $(2 \mathrm{~T}) / 2$. Then, Hölder's inequality gives

$$
B f(x) \leq \frac{c_{M}^{\prime}}{\max \left\{1,|x|^{d}\right\}}\|f\|_{L^{p}(v)}\left\|v^{-\frac{1}{p}} e^{-\frac{|y|^{2}}{4 s_{0}}}\right\|_{p^{\prime}}<\infty .
$$

So, we will have $\|B f\|_{L^{p}(u)} \lesssim\|f\|_{L^{p}(v)}$ if we choose any $u(x) \leq 1$. We now pass to the operator $A f(x)$. Since $|y| \leq M \max \{|x|, 1\}$, an elementary computation slicing into dyadic shells (of radii $2^{j} \sqrt{s}, j \in \mathbb{N}$ ) gives

$$
A f(x) \leq \sup _{0<t<a} \int_{|y| \leq M \max \{|x|, 1\}}|f(y)| \frac{e^{-\frac{|x-y|^{2}}{4 s}}}{(4 \pi s)^{d / 2}} d y \lesssim \mathcal{M}_{M}^{\text {loc }} f(x),
$$

where $\mathcal{M}_{M}^{\text {loc }} f$ is the local Hardy-Littlewood maximal function defined in (2.1). Thus by Theorem [2.1, if we set $\sigma_{0}=\sigma_{0}\left(a, \mathrm{~T}^{*}\right)=1 / M^{2} \in(0,1)$, then for every $\sigma<\sigma_{0}$ there exists a weight $U$ such that $\|A f\|_{L^{p}(U)} \lesssim\|f\|_{L^{p}(v)}$ and $\left\|U^{-\frac{\sigma}{p}} e^{-\frac{|y|^{2}}{4 s}}\right\|_{p^{\prime}}<\infty$ for all $s<\operatorname{th}(2 \mathrm{~T}) / 2$. Finally, to combine the estimates for $A f$ and $B f$ we can just take

$$
u(x)=\min \{U(x), 1\}
$$

which satisfies the required properties.

Remark 3.6. In the previous proof we chose $\sigma_{0}\left(a, \mathrm{~T}^{*}\right)=1 / M^{2}$, with $M$ so that $\left(\frac{M}{M-1}\right)^{3} \frac{\text { th } 2 a}{2}<\mathrm{T}^{*}$. In general, we cannot let $M \searrow 1$ (and hence $\sigma_{0} \nearrow 1$ ), but we could always do so if we start with a parameter $a$ sufficiently small (depending on $\left.T^{*}\right)$.

\section{The Poisson equation for the Hermite operator}

4.1. Kernel estimates. We denote by $p_{t}(x, y)$ the kernel of the operator $e^{-t \sqrt{L}}$. By the subordination formula (1.5), and using the explicit expression for $h_{t}(x, y)$ in (3.1), the kernel can be written as

$$
\begin{aligned}
p_{t}(x, y) & =\frac{t}{\sqrt{4 \pi}} \int_{0}^{\infty} e^{-\frac{t^{2}}{4 u}} h_{u}(x, y) \frac{d u}{u^{3 / 2}} \\
& =\frac{t}{(4 \pi)^{\frac{d+1}{2}}} \int_{0}^{1} \frac{e^{-\frac{t^{2}}{2 \ln \frac{1+s}{1-s}}}\left(1-s^{2}\right)^{\frac{d}{2}-1} e^{-\frac{1}{4}\left(\frac{|x-y|^{2}}{s}+s|x+y|^{2}\right)}}{s^{\frac{d}{2}}\left(\frac{1}{2} \ln \frac{1+s}{1-s}\right)^{3 / 2}} d s
\end{aligned}
$$

after the change of variables $s=\operatorname{th} u$ (or equivalently $u=\frac{1}{2} \ln \frac{1+s}{1-s}$ ). These long expressions are difficult to handle, but we obtain here very precise decay estimates which will be enough for our purposes. The next two lemmas are key results in this section.

Lemma 4.1. Given $t>0$ and $x \in \mathbb{R}^{d}$, there exists some $C_{t, x}>0$ such that

$$
\frac{C_{t, x}^{-1} e^{-\frac{|y|^{2}}{2}}}{(1+|y|)^{\frac{d}{2}}[\ln (e+|y|)]^{\frac{3}{2}}} \leq p_{t}(x, y) \leq \frac{C_{t, x} e^{-\frac{|y|^{2}}{2}}}{(1+|y|)^{\frac{d}{2}}[\ln (e+|y|)]^{\frac{3}{2}}}, \quad \forall y \in \mathbb{R}^{d} .
$$

Proof. We may assume that $|y| \geq M \max \{|x|, 1\}$, for a fixed sufficiently large $M$, since otherwise $y$ would belong to a compact set, and upper and lower bounds with a constant $C_{t, x}$ would be obvious. 
We start with the upper bound. In the integral expression for $p_{t}(x, y)$ in (4.1), we first look at the range $0<s<1 / 2$, namely

$$
\begin{aligned}
I_{0} & =t \int_{0}^{1 / 2} \frac{e^{-\frac{t^{2}}{2 \ln \frac{1+s}{1-s}}}\left(1-s^{2}\right)^{\frac{d}{2}-1} e^{-\frac{1}{4}\left(a^{2} s+\frac{b^{2}}{s}\right)}}{s^{\frac{d}{2}}\left(\ln \frac{1+s}{1-s}\right)^{3 / 2}} d s \\
& \lesssim t \int_{0}^{1 / 2} \frac{e^{-c t^{2} / s} e^{-\frac{1}{4}\left(a^{2} s+\frac{b^{2}}{s}\right)}}{s^{\frac{d+3}{2}}} d s,
\end{aligned}
$$

where we have used the elementary estimate

$$
\ln \left(\frac{1+s}{1-s}\right)=\ln \left(1+\frac{2 s}{1-s}\right) \approx s, \quad s \in[0,1 / 2],
$$

and the notation

$$
a=|x+y| \quad \text { and } \quad b=|x-y| .
$$

We turn to estimating the crucial exponential factor

$$
\exp \left(-\frac{1}{4}\left(s a^{2}+\frac{b^{2}}{s}\right)\right)
$$

If $0<s<1 / 2$, by monotonocity we have $s+\frac{1}{s} \geq \frac{5}{2}$, while the assumption $|y| \geq M|x|$ implies that $a^{2}, b^{2} \geq\left(\frac{M-1}{M}\right)^{2}|y|^{2}$. Thus, the exponential term in (4.5) is controlled by

$$
\exp \left(-\frac{1}{4}\left(s a^{2}+\frac{b^{2}}{s}\right)\right) \leq \exp \left(-\frac{5}{8}\left(\frac{M-1}{M}\right)^{2}|y|^{2}\right) \leq \exp \left(-\frac{6}{10}|y|^{2}\right),
$$

if $M$ is chosen large enough. Inserting this bound into (4.3) and changing variables $v=t^{2} / s$ in the remaining integral we obtain

$$
I_{0} \lesssim e^{-\frac{6}{10}|y|^{2}} t^{-d} \int_{2 t^{2}}^{\infty} e^{-c v} v^{\frac{d+1}{2}} \frac{d v}{v} \leq c^{\prime} t^{-d} e^{-\frac{6}{10}|y|^{2}}
$$

Thus, this part has a better decay than the right hand side of (4.2).

We now pass to the range $1 / 2 \leq s<1$, which disregarding irrelevant terms is given by the integral

$$
I_{1}=t \int_{\frac{1}{2}}^{1} \frac{(1-s)^{\frac{d}{2}-1}}{\left(\ln \frac{1}{1-s}\right)^{\frac{3}{2}}} e^{-\frac{1}{4}\left(s a^{2}+\frac{b^{2}}{s}\right)} d s .
$$

Here we need a finer estimate on the exponential (4.5). Completing squares, we can write the exponent as

$$
s a^{2}+\frac{b^{2}}{s}=\left(\sqrt{s} a-\frac{b}{\sqrt{s}}\right)^{2}+2 a b=\frac{a^{2}}{s}\left(s-\frac{b}{a}\right)^{2}+2 a b .
$$

Since $a b=|x+y||x-y| \geq(y+x) \cdot(y-x)=|y|^{2}-|x|^{2}$, we have

$$
e^{-\frac{1}{4}\left(s a^{2}+\frac{b^{2}}{s}\right)} \leq e^{\frac{|x|^{2}-|y|^{2}}{2}} e^{-\frac{a^{2}}{4 s}\left(s-\frac{b}{a}\right)^{2}} .
$$

We shall estimate the last exponential as follows. First note that, by the triangle inequality, $|b-a|=|| x-y|-| x+y|| \leq 2|x|$. Therefore

$$
\left|s-\frac{b}{a}\right| \geq|1-s|-\left|\frac{b}{a}-1\right| \geq 1-s-\frac{2|x|}{a} .
$$


So when we consider the range $1 / 2 \leq s<1-\frac{4|x|}{a}$, we have $\left|s-\frac{b}{a}\right| \geq(1-s) / 2$, and hence

$$
\begin{aligned}
I_{10}=\int_{\frac{1}{2}}^{1-\frac{4|x|}{a}} \cdots & \leq t e^{\frac{|x|^{2}-|y|^{2}}{2}} \int_{1 / 2}^{1-\frac{4|x|}{a}} \frac{(1-s)^{\frac{d}{2}-1}}{\left(\ln \frac{1}{1-s}\right)^{\frac{3}{2}}} e^{-\frac{a^{2}(1-s)^{2}}{16}} d s \\
& \leq t e^{\frac{|x|^{2}-|y|^{2}}{2}} a^{-d / 2} \int_{0}^{a / 2} \frac{v^{\frac{d}{2}-1}}{\left(\ln \frac{a}{v}\right)^{\frac{3}{2}}} e^{-\frac{v^{2}}{16}} d v,
\end{aligned}
$$

where in the last line we have changed variables $v=a(1-s)$ and have slightly enlarged the set of integration. Now, it is easy to see that, in the last integral, the main contribution happens when $v \approx 1$, and hence the integral is controlled by $c(\ln a)^{-3 / 2}$. Since $a=|x+y| \geq \frac{M-1}{M}|y|$, this part meets the required bound on the right hand side of (4.2).

It remains to consider the range $1-4|x| / a \leq s<1$, in which we shall disregard the last exponential in (4.8). That is,

$$
I_{11}=\int_{1-\frac{4|x|}{a}}^{1} \cdots \leq t e^{\frac{|x|^{2}-|y|^{2}}{2}} \int_{1-\frac{4|x|}{a}}^{1} \frac{(1-s)^{\frac{d}{2}-1}}{\left(\ln \frac{1}{1-s}\right)^{\frac{3}{2}}} d s \leq t e^{\frac{|x|^{2}-|y|^{2}}{2}} \int_{0}^{c \mid \frac{|x|}{|y|}} \frac{u^{\frac{d}{2}-1}}{\left(\ln \frac{1}{u}\right)^{\frac{3}{2}}} d u,
$$

where in the last step we changed varibles $u=1-s$ and used $a=|x+y| \geq \frac{M-1}{M}|y|$ (so one can choose $c=4 M /(M-1)$ ). Now, in this range of integration we have $\log (1 / u) \geq \log \frac{|y|}{c|x|}$, so the right hand side can be estimated by

$$
I_{11} \lesssim t e^{\frac{|x|^{2}-|y|^{2}}{2}}\left(\frac{|x|}{|y|}\right)^{d / 2}\left[\log \frac{|y|}{c|x|}\right]^{-\frac{3}{2}}
$$

If say $|x| \leq \sqrt{M} / c$, using $|y| \geq M$ we have $\log \frac{|y|}{c|x|} \geq \frac{1}{2} \log |y|$. If $|x| \geq \sqrt{M} / c$ (and $M$ is sufficiently large) we can use the elementary inequality

$$
\log |y|=\log \frac{|y|}{c|x|}+\log (c|x|) \leq \log \frac{|y|}{c|x|} \cdot \log (c|x|),
$$

which implies $\log \frac{|y|}{c|x|} \geq \log |y| / \log (c|x|)$. Inserting these estimates of the logarithmic term into (4.9) we finally obtain

$$
I_{11} \lesssim t c_{x} e^{-\frac{|y|^{2}}{2}}|y|^{-d / 2}[\log |y|]^{-\frac{3}{2}},
$$

with constant $c_{x}=e^{\frac{|x|^{2}}{2}}(1+|x|)^{d / 2}[\log (e+|x|)]^{3 / 2}$. Combining the bounds for $I_{10}$ and $I_{11}$ we conclude that

$$
I_{1} \leq t c_{x} e^{\frac{-|y|^{2}}{2}}(1+|y|)^{-d / 2}[\log (e+|y|)]^{-3 / 2},
$$

when $|y| \geq M(|x| \vee 1)$, with the same value of $c_{x} 3$

We finally prove the lower bound. The main contribution for the integral defining $p_{t}(x, y)$ will appear when $1-s \approx 1 /|y|$. To show this, notice that since $\frac{1+s}{1-s}$ is increasing when $1 / 2 \leq s<1$, we can bound

$$
\exp \left(-\frac{t^{2}}{2 \ln \frac{1+s}{1-s}}\right) \geq \exp \left(-\frac{t^{2}}{2 \ln 3}\right) .
$$

\footnotetext{
${ }^{3}$ This value of $c_{x}$ will play a role later.
} 
Thus

$$
p_{t}(x, y) \geq c_{t} \int_{1-\frac{1}{|y|}}^{1} \frac{(1-s)^{\frac{d}{2}-1}}{\left(\ln \frac{2}{1-s}\right)^{\frac{3}{2}}} e^{-\frac{1}{4}\left(s a^{2}+\frac{b^{2}}{s}\right)} d s,
$$

where as before $a=|x+y|$ and $b=|x-y|$. Changing variables $1-s=u$, we are led to consider

$$
I=\int_{0}^{1 /|y|} \frac{u^{\frac{d}{2}-1}}{\left(\ln \frac{2}{u}\right)^{\frac{3}{2}}} e^{-\frac{1}{4}\left((1-u) a^{2}+\frac{b^{2}}{1-u}\right)} d u .
$$

This time we write the terms in the exponential expression as

$$
(1-u) a^{2}+\frac{b^{2}}{1-u}=a^{2}+b^{2}-\frac{\left(a^{2}-b^{2}\right) u}{1-u}+\frac{a^{2} u^{2}}{1-u} .
$$

Thus, since $a^{2}+b^{2}=2\left(|x|^{2}+|y|^{2}\right)$ and $a^{2}-b^{2}=4 x \cdot y$, we see that

$$
\begin{aligned}
\exp \left(-\frac{1}{4}\left((1-u) a^{2}+\frac{b^{2}}{1-u}\right)\right) & =\exp \left(-\frac{|x|^{2}+|y|^{2}}{2}\right) \exp \left(\frac{u}{1-u} x \cdot y\right) \exp \left(-\frac{a^{2} u^{2}}{4(1-u)}\right) \\
& \geq \exp \left(-\frac{|x|^{2}+|y|^{2}}{2}\right) \exp \left(-\frac{|x||y| u}{1-u}\right) \exp \left(-2|y|^{2} u^{2}\right),
\end{aligned}
$$

where in the last line we used that $1-u \geq 1 / 2$ and $a=|x+y| \leq 2|y|$. Thus,

$$
I \geq e^{-\frac{|x|^{2}+|y|^{2}}{2}} \int_{0}^{1 /|y|} \frac{u^{\frac{d}{2}-1}}{\left(\ln \frac{2}{u}\right)^{\frac{3}{2}}} e^{-2|x||y| u} e^{-2|y|^{2} u^{2}} d u .
$$

Finally, since $u|y| \leq 1$, we can bound from below:

$$
I \geq e^{-\frac{|x|^{2}+|y|^{2}}{2}} e^{-2|x|} e^{-2} \int_{0}^{1 /|y|} \frac{u^{\frac{d}{2}}}{\left(\ln \frac{2}{u}\right)^{\frac{3}{2}}} \frac{d u}{u} \gtrsim e^{-\frac{|x|^{2}}{2}-2|x|} \frac{e^{-\frac{|y|^{2}}{2}}}{(1+|y|)^{\frac{d}{2}}[\ln (e+|y|)]^{\frac{3}{2}}},
$$

as we wished to prove.

The previous lemma essentially suffices for the proof of Theorem 1.1. For Theorem 1.3. however, a more precise bound is needed. Below we write $p_{t}^{\Delta}(x-y)$ for the usual Poisson kernel

$$
p_{t}^{\Delta}(x-y)=\frac{c_{d} t}{\left(t^{2}+|x-y|^{2}\right)^{\frac{d+1}{2}}} .
$$

Lemma 4.2. There exists a constant $\gamma \geq 2$ such that

$$
p_{t}(x, y) \leq C_{x}\left[p_{t}^{\Delta}(x-y) e^{-\frac{|y|^{2}}{2}} \chi_{\{|y| \leq \gamma \max \{|x|, 1\}\}}+\frac{t e^{-\frac{|y|^{2}}{2}}}{(1+|y|)^{\frac{d}{2}}[\ln (e+|y|)]^{\frac{3}{2}}}\right],
$$

where $C_{x}=C(1+|x|)^{d} e^{|x|^{2} / 2}$, for some constant $C>0$.

Proof. As before, we split the integral in (4.1), which defines $p_{t}(x, y)$ as

$$
p_{t}(x, y)=\int_{0}^{\frac{1}{2}} \ldots+\int_{\frac{1}{2}}^{1} \ldots \leq I_{0}+I_{1}
$$

with $I_{0}$ and $I_{1}$ given in (4.3) and (4.7), respectively. We have already shown in (4.10) that

$$
I_{1} \lesssim c_{x} \frac{t e^{-\frac{|y|^{2}}{2}}}{(1+|y|)^{\frac{d}{2}}[\ln (e+|y|)]^{\frac{3}{2}}}, \quad \text { if } \quad|y| \geq M(|x| \vee 1)
$$


with $c_{x} \leq C_{x}$ (see footnote 3). For $|y| \leq M(|x| \vee 1$ ) we shall use a cruder bound: keeping the notation in (4.4) and disregarding the last exponential factor in (4.8) we see that $\exp \left(-\frac{1}{4}\left(s a^{2}+\frac{b^{2}}{s}\right)\right) \leq e^{\frac{|x|^{2}}{2}} e^{-\frac{|y|^{2}}{2}}$. Inserting this into (4.7) we obtain

$$
I_{1} \leq t e^{\frac{|x|^{2}}{2}} e^{-\frac{|y|^{2}}{2}} \int_{\frac{1}{2}}^{1} \frac{(1-s)^{\frac{d}{2}-1}}{\left(\ln \frac{1}{1-s}\right)^{\frac{3}{2}}} d s \leq C_{x} \frac{t e^{-\frac{|y|^{2}}{2}}}{(1+|y|)^{\frac{d}{2}}[\ln (e+|y|)]^{\frac{3}{2}}} .
$$

We now turn to estimate $I_{0}$, for which we need a slightly different argument to introduce $p_{t}^{\Delta}$. Starting from (4.6), we can write, for a small $\eta>0$ (to be determined),

$$
I_{0} \leq t \int_{2}^{\infty} e^{-\left(c t^{2}+\frac{\eta}{4} b^{2}\right) v} e^{-\frac{1}{4}\left(\frac{a^{2}}{v}+b^{2}(1-\eta) v\right)} v^{\frac{d+1}{2}} \frac{d v}{v} .
$$

Now, if $|y| \geq M \max \{|x|, 1\}$, we use as before that $a^{2}, b^{2} \geq\left(\frac{M-1}{M}\right)^{2}|y|^{2}$ and the monotonicity of $\frac{1}{v}+v$ to obtain

$$
e^{-\frac{1}{4}\left(\frac{a^{2}}{v}+b^{2}(1-\eta) v\right)} \leq e^{-\frac{1}{4}(1-\eta)\left(\frac{M-1}{M}\right)^{2}\left(\frac{1}{v}+v\right)|y|^{2}} \leq e^{-\frac{5}{8}(1-\eta)\left(\frac{M-1}{M}\right)^{2}|y|^{2}} .
$$

So, setting $\eta=1 / M$ and fixing $M$ large enough such that $\frac{5}{8}\left(\frac{M-1}{M}\right)^{3} \geq \frac{6}{10}$, we conclude that

$$
\begin{aligned}
I_{0} & \leq c_{M} t e^{-\frac{6}{10}|y|^{2}} \int_{2}^{\infty} e^{-\left(c t^{2}+\frac{\eta}{4} b^{2}\right) v} v^{\frac{d+1}{2}} \frac{d v}{v} \\
& \leq c_{M}^{\prime} \frac{t e^{-\frac{6}{10}|y|^{2}}}{\left(t^{2}+|x-y|^{2}\right)^{\frac{d+1}{2}}} \leq c_{M}^{\prime \prime} \frac{t e^{-\frac{6}{10}|y|^{2}}}{|y|^{d+1}},
\end{aligned}
$$

using in the last step the assumption $|y| \geq M \max \{|x|, 1\}$. This can clearly be absorbed into the right hand side of (4.12).

So we are left with $I_{0}$ in the region $|y| \leq M \max \{|x|, 1\}$. We now write

$$
\begin{aligned}
I_{0} & \leq t \int_{2}^{\infty} e^{-\frac{1}{4}\left(c t^{2}+b^{2}\right) v} e^{-\frac{a^{2}}{4 v}} v^{\frac{d+1}{2}} \frac{d v}{v} \\
& \lesssim \frac{t}{\left(c t^{2}+b^{2}\right)^{\frac{d+1}{2}}} \int_{\frac{c t^{2}+b^{2}}{2}}^{\infty} e^{-u} e^{-\frac{a^{2}\left(c t^{2}+b^{2}\right)}{16 u}} u^{\frac{d+1}{2}} \frac{d u}{u},
\end{aligned}
$$

where we have changed variables $u=\left(c t^{2}+b^{2}\right) v / 4$. In the last integral we can disregard $t$ and estimate it crudely by

$$
J=\int_{0}^{\infty} e^{-u} e^{-\frac{a^{2} b^{2}}{16 u}} u^{\frac{d+1}{2}} \frac{d u}{u} .
$$

This integral is not difficult to estimate by hand, but we can also quote the literature, since it resembles a Bessel potential kernel

$$
G_{\alpha}(x)=c_{\alpha, d} \int_{0}^{\infty} e^{-u} e^{-\frac{|x|^{2}}{4 u}} u^{\frac{\alpha-d}{2}} \frac{d u}{u}
$$

[16, p. 132]. Indeed, modulo a multiplicative constant $J=c_{d} G_{2 d+1}(a b / 2)$ and from well-known estimates of the Bessel potential kernel (see e.g. [3. p. 417]) we obtain

$$
J \lesssim(1+a b)^{\frac{d}{2}} e^{-\frac{a b}{2}} .
$$

Now, $a b \geq|\langle x+y, x-y\rangle| \geq-|x|^{2}+|y|^{2}$, so using the assumption $|y| \leq M|x|$ we obtain

$$
J \lesssim e^{\frac{|x|^{2}}{2}} e^{-\frac{|y|^{2}}{2}}(1+|x+y||x-y|)^{\frac{d}{2}} \leq c_{M}(1+|x|)^{d} e^{\frac{|x|^{2}}{2}} e^{-\frac{|y|^{2}}{2}} .
$$


Inserting this into (4.13) we obtain the bound asserted in the statement of the lemma.

4.2. Proof of Theorem 1.1 for the Hermite-Poisson equation. As we did before, the result will follow directly from the next three propositions. The first one is a direct consequence of the kernel estimates in Lemma 4.1 .

Proposition 4.3. The following properties are equivalent:

(i) $\int_{\mathbb{R}^{d}} p_{t}(x, y)|f(y)| d y<\infty$, for all $t>0$ and $x \in \mathbb{R}^{d}$;

(ii) $\int_{\mathbb{R}^{d}} p_{t_{0}}\left(x_{0}, y\right)|f(y)| d y<\infty$, for some $t_{0}>0$ and some $x_{0} \in \mathbb{R}^{d}$;

(iii) $\int_{\mathbb{R}^{d}}|f(y)| e^{-\frac{|y|^{2}}{2}}(1+|y|)^{-\frac{d}{2}}[\ln (e+|y|)]^{-\frac{3}{2}} d y<\infty$.

For the smoothness of the solution one argues similarly to Proposition 3.4.

Proposition 4.4. If $f$ satisfies the conditions in Proposition 4.3, then

$$
u(t, x)=\int_{\mathbb{R}^{d}} p_{t}(x, y) f(y) d y \in C^{\infty}\left((0, \infty) \times \mathbb{R}^{d}\right) .
$$

Proof. It suffices to prove that, for each $x$ and $t$,

$$
\int_{\mathbb{R}^{d}}\left|\partial_{t}^{L} \Delta_{x}^{M}\left[p_{t}(x, y)\right]\right||f(y)| d y<\infty, \quad \forall L, M \geq 0 .
$$

Since the Poisson kernel satisfies $\Delta_{x} p_{t}(x, y)=|x|^{2} p_{t}(x, y)-\partial_{t t}\left[p_{t}(x, y)\right]$, we can assume $M=0$. Now, taking derivatives with respect to $t$ in the explicit formula (4.1), matters are reduced to checking that, for each $\ell \geq 0$,

$$
\int_{0}^{1} \frac{e^{-\frac{t^{2}}{2 \ln \frac{1+s}{1-s}}}\left(1-s^{2}\right)^{\frac{d}{2}-1} e^{-\frac{1}{4}\left(\frac{|x-y|^{2}}{s}+s|x+y|^{2}\right)}}{s^{\frac{d}{2}}\left(\frac{1}{2} \ln \frac{1+s}{1-s}\right)^{3 / 2+\ell}} d s<\infty .
$$

But the same proof we gave in Lemma 4.1 shows that this is equivalent to

$$
\int_{\mathbb{R}^{d}}|f(y)| e^{-\frac{|y|^{2}}{2}}(1+|y|)^{-\frac{d}{2}}[\ln (e+|y|)]^{-\left(\frac{3}{2}+\ell\right)} d y<\infty,
$$

which holds under the assumptions of the proposition.

For the pointwise limits we shall also need the estimates in Lemma 4.2 .

Proposition 4.5. If $f$ satisfies the conditions in Proposition 4.3, then

$$
\lim _{t \rightarrow 0^{+}} e^{-t \sqrt{L}} f(x)=f(x), \quad \text { a.e. } x \in \mathbb{R}^{d} .
$$

Proof. We argue as in the proof of Proposition 3.5. We shall show (4.16) for a.e. $|x| \leq n$ and every fixed $n \in \mathbb{N}$. Split

$$
f=f \chi_{\{|y| \leq \gamma n\}}+f \chi_{\{|y|>\gamma n\}}=f_{1}+f_{2}
$$

( $\gamma$ is the constant in Lemma 4.2). Using Lemma 4.2 we see that, for every $|x| \leq n$,

$$
\left|e^{-t \sqrt{L}} f_{2}(x)\right| \leq C_{n_{0}} t \int_{\mathbb{R}^{d}} \frac{|f(y)| e^{-\frac{|y|^{2}}{2}} d y}{(1+|y|)^{\frac{d}{2}}[\ln (e+|y|)]^{\frac{3}{2}}} .
$$

Since the last integral is finite we obtain $\lim _{t \rightarrow 0} e^{-t \sqrt{L}} f_{2}(x)=0$ for all $|x| \leq n$. 
On the other hand, $f_{1} \in L^{1}\left(\mathbb{R}^{d}\right)$, so the standard pointwise convergence of Hermite-Poisson integrals of $L^{1}$ functions gives

$$
\lim _{t \rightarrow 0} e^{-t \sqrt{L}} f_{1}(x)=f(x), \quad \text { a.e. }|x| \leq n .
$$

4.3. Proof of Theorem 1.3. Let $v \in D_{p}^{\text {Pois }}(L)$, that is,

$$
\|v\|_{D_{p}}:=\left\|v^{-\frac{1}{p}} \varphi\right\|_{L^{p^{\prime}\left(\mathbb{R}^{d}\right)}}<\infty
$$

where we denote $\varphi(y)=e^{-\frac{|y|^{2}}{2}}(1+|y|)^{-\frac{d}{2}}[\ln (e+|y|)]^{-\frac{3}{2}}$. For every $a>0$ and $\sigma<1$, we must show the boundedness of the maximal operator

$$
P_{a}^{*} f(x)=\sup _{0<t<a} \int_{\mathbb{R}^{d}} p_{t}(x, y)|f(y)| d y
$$

from $L^{p}(v) \rightarrow L^{p}(u)$ for some other weight $u$ such that $u^{\sigma} \in D_{p}$.

We again use Lemma 4.2 to split the maximal function as

$$
\begin{aligned}
P_{a}^{*} f(x) \leq & C_{x} \sup _{0<t<a} \int_{|y| \leq \gamma \max \{|x|, 1\}} p_{t}^{\Delta}(x, y)|f(y)| e^{-\frac{|y|^{2}}{2}} d y \\
& +C_{x} a \int_{\mathbb{R}^{d}}|f(y)| \varphi(y) d y \\
= & P_{a}^{*, 0} f(x)+P_{a}^{*, 1} f(x),
\end{aligned}
$$

where we recall that $C_{x}=c(1+|x|)^{d} \exp \left(|x|^{2} / 2\right)$. Clearly, by Hölder's inequality,

$$
P_{a}^{*, 1} f(x) \leq C_{x} a\|f\|_{L^{p}(v)}\|v\|_{D_{p}},
$$

so we will have $\left\|P_{a}^{*, 1} f\right\|_{L^{p}(u)} \lesssim\|f\|_{L^{p}(v)}$ provided we choose

$$
u(x) \leq u_{1}(x):=\frac{1}{C_{x}^{p}(1+|x|)^{d+1}}=c^{\prime} e^{-\frac{p}{2}|x|^{2}}(1+|x|)^{-d(p+1)-1} .
$$

Observe that $\left\|u_{1}^{-\frac{\sigma}{p}} \varphi\right\|_{p^{\prime}}<\infty$ for every $\sigma<1$.

On the other hand, when $|y| \leq \gamma \max \{|x|, 1\}$, a standard argument slicing the integral into shells gives

$$
P_{a}^{*, 0} f(x) \lesssim C_{x} \sup _{t>0} \int_{|y| \leq \gamma \max \{|x|, 1\}} \frac{t|f(y)| e^{-\frac{|y|^{2}}{2}}}{(t+|x-y|)^{d+1}} d y \lesssim C_{x} \mathcal{M}_{\gamma}^{\text {loc }}\left(f e^{-\frac{|y|^{2}}{2}}\right)(x),
$$

with $\mathcal{M}_{\gamma}^{\text {loc }}$ defined in (2.1). Now, the new function $\tilde{f}(y)=f(y) e^{-\frac{|y|^{2}}{2}} \in L^{p}(\tilde{v})$ for the weight $\tilde{v}(y)=v(y) e^{\frac{p}{2}|y|^{2}}$. By (4.19), this weight satisfies

$$
\left\|\tilde{v}^{-\frac{1}{p}} e^{-\varepsilon|y|^{2}}\right\|_{p^{\prime}}=\left\|v^{-\frac{1}{p}} e^{-\left(\frac{1}{2}+\varepsilon\right)|y|^{2}}\right\|_{p^{\prime}}<\infty, \quad \forall \varepsilon>0,
$$

so we can apply Theorem 2.1 to find, for each $\sigma<1$, a weight $\tilde{U}$ such that

$$
\left\|\mathcal{M}_{\gamma+1}^{\text {loc }} \tilde{f}\right\|_{L^{p}(\tilde{U})} \lesssim\|\tilde{f}\|_{L^{p}(\tilde{v})}=\|f\|_{L^{p}(v)} \quad \text { and } \quad\left\|\tilde{U}^{-\frac{\sigma}{p}} e^{-\varepsilon|y|^{2}}\right\|_{p^{\prime}}<\infty, \quad \forall \varepsilon>0 .
$$

Then, setting

we see that

$$
u_{0}(x)=\frac{\tilde{U}(x)}{C_{x}^{p}}=c^{\prime} \tilde{U}(x) e^{-\frac{p}{2}|x|^{2}}(1+|x|)^{-d p},
$$

$\left\|P_{a}^{*, 0} f\right\|_{L^{p}\left(u_{0}\right)} \lesssim\|f\|_{L^{p}(v)} \quad$ and $\quad\left\|u_{0}^{-\frac{\sigma}{p}} \varphi\right\|_{p^{\prime}} \leq\left\|\tilde{U}^{-\frac{\sigma}{p}}(1+|x|)^{d \sigma} e^{-\frac{1-\sigma}{2}|x|^{2}}\right\|_{p^{\prime}}<\infty$. 
Finally, we can combine the estimates for $P_{a}^{*, 0} f$ and $P_{a}^{*, 1} f$ by taking

$$
u(x)=\min \left\{u_{0}(x), u_{1}(x)\right\},
$$

which satisfies the required properties.

\section{The Perturbed Laplacian $L=-\Delta+R$ With $R>0$}

This operator can often be seen as a toy model for the study of the Hermite operator. We think that the results obtained in this case are interesting in themselves, so we present them here in some detail. Throughout this section, $R>0$ is fixed.

5.1. Heat equation for $L=-\Delta+R$. This case is a direct consequence of the corresponding results for the classical heat equation, since

$$
e^{-t L} f(x)=e^{-t R} e^{t \Delta} f(x)=e^{-t R}\left(W_{t} * f\right)(x),
$$

where $W_{t}(x)=(4 \pi t)^{-d / 2} \exp \left(-|x|^{2} /(4 t)\right)$ is the Gauss-Weierstrass kernel. Indeed, the factor $e^{-t R}$ is irrelevant for the questions asked here, so the characterizations will be the same for both operators $L=-\Delta+R$ and $L=-\Delta$.

We remark that for the classical heat equation, both Theorems 1.1 and 1.3 are known or can be obtained with the same methods we carried out in $§ 3$, so we do not include the proofs here. For example, the results stated in Theorem 1.1 can be found in [21, pp. 64-67] or [22] or, alternatively, can be proved arguing exactly as in 3.2 (with a few obvious modifications). The same applies to Theorem 1.3 , basically reading line by line the proof given in $\$ 3.3$ or checking the reference 7 . (our formulation is slightly more general, but the ideas are similar).

5.2. Poisson equation for $L=-\Delta+R$. This case requires a formal proof, since it cannot be deduced from the classical setting. In fact, the characterizing conditions are different for $-\Delta$ and $-\Delta+R$; see (1.3) and Table 1, Our method will be similar to the one presented in $\varangle$ for the Hermite case.

The Poisson kernel in this case is of convolution type $p_{t}(x, y)=p_{t}(x-y)$. From the subordination formula (1.5) and the factorization in (5.1), the kernel takes the form

$$
\begin{aligned}
p_{t}(x) & =\frac{t}{(4 \pi)^{\frac{d+1}{2}}} \int_{0}^{\infty} e^{-\frac{t^{2}+|x|^{2}}{4 u}} e^{-u R} u^{-\frac{d+3}{2}} d u \\
& =\frac{t}{\left[\pi\left(t^{2}+|x|^{2}\right)\right]^{\frac{d+1}{2}}} \int_{0}^{\infty} e^{-v} e^{-\frac{R\left(t^{2}+|x|^{2}\right)}{4 v}} v^{\frac{d+1}{2}} d v
\end{aligned}
$$

where in the last line we have changed variables $v=\left(t^{2}+|x|^{2}\right) /(4 u)$. One recognizes in this expression the standard Poisson kernel $p_{t}^{\Delta}(x)$ and the Bessel potential kernel defined in (4.14), so that we can write

$$
p_{t}(x)=c_{d} p_{t}^{\Delta}(x) G_{2 d+1}\left(\sqrt{R\left(t^{2}+|x|^{2}\right)}\right),
$$

for a suitable constant $c_{d}$. Since we only care about approximate expressions, using the asymptotics of $G_{\alpha}(x)$ mentioned already in 4.1 we obtain

$$
p_{t}(x-y) \approx \frac{t(1+\sqrt{R}(t+|x-y|))^{\frac{d}{2}} e^{-\sqrt{R\left(t^{2}+|x-y|^{2}\right)}}}{(t+|x-y|)^{d+1}} .
$$

To derive a less complicated expression for this kernel we need an elementary lemma. 
Lemma 5.1. For every $t>0$ and $x \in \mathbb{R}^{d}$ we have

$$
c_{1}(t, x) e^{-\sqrt{R\left(1+|y|^{2}\right)}} \leq e^{-\sqrt{R\left(t^{2}+|x-y|^{2}\right)}} \leq c_{2}(x) e^{-\sqrt{R\left(1+|y|^{2}\right)}}, \quad \forall y \in \mathbb{R}^{d},
$$

for some $c_{1}(t, x)>0$ and $c_{2}(x)=c_{R} \exp (\sqrt{R}|x|)$.

Proof. For the left inequality notice that

$$
\sqrt{t^{2}+|x-y|^{2}} \leq t+|x|+|y| \leq t+|x|+\sqrt{1+|y|^{2}} .
$$

Hence

$$
e^{-\sqrt{R\left(t^{2}+|x-y|^{2}\right)}} \geq c_{1}(t, x) e^{-\sqrt{R\left(1+|y|^{2}\right)}}
$$

with $c_{1}(t, x)=\exp (-\sqrt{R}(t+|x|))$.

To prove the right inequality in (5.5), assume first that $|y| \geq 1$. Then

$$
e^{-\sqrt{R} \sqrt{t^{2}+|x-y|^{2}}} \leq e^{-\sqrt{R}|x-y|} \leq e^{\sqrt{R}|x|} e^{-\sqrt{R}|y|} .
$$

Now, using first the assumption $|y| \geq 1$, followed by the elementary inequality $1+\frac{a}{2} \geq \sqrt{1+a}$, we obtain

$$
|y| \geq|y|\left(1+\frac{1}{2|y|^{2}}\right)-\frac{1}{2} \geq|y| \sqrt{1+\frac{1}{|y|^{2}}}-\frac{1}{2}=\sqrt{1+|y|^{2}}-\frac{1}{2} .
$$

Inserting this into (5.6) we obtain

$$
e^{-\sqrt{R} \sqrt{t^{2}+|x-y|^{2}}} \leq e^{\sqrt{R}\left(|x|+\frac{1}{2}\right)} e^{-\sqrt{R\left(1+|y|^{2}\right)}} .
$$

Finally, if $|y| \leq 1$ one trivially has

$$
e^{-\sqrt{R} \sqrt{t^{2}+|x-y|^{2}}} \leq 1=e^{\sqrt{R\left(1+|y|^{2}\right)}} e^{-\sqrt{R\left(1+|y|^{2}\right)}} \leq e^{\sqrt{2 R}} e^{-\sqrt{R\left(1+|y|^{2}\right)}} .
$$

Combining the last two estimates we obtain (5.5) with $c_{2}(x)=\exp (\sqrt{R}(|x|+2))$.

Lemma 5.2. The following inequality holds for every $t>0$ and $x, y \in \mathbb{R}^{d}$ :

$$
p_{t}(x-y) \leq C_{x}\left[\max \{t, 1\}^{\frac{d}{2}} \frac{p_{t}^{\Delta}(x-y)}{e^{\sqrt{R\left(1+|y|^{2}\right)}}} \chi_{\{|y| \leq 2 \max \{1,|x|\}}+\frac{t e^{-\sqrt{R\left(1+|y|^{2}\right)}}}{(1+|y|)^{\frac{d}{2}+1}}\right] \text {, }
$$

where $C_{x}=c_{R}(1+|x|)^{d / 2} e^{\sqrt{R}|x|}$.

Proof. The proof is an easy estimation combining (5.4) with Lemma 5.1

Theorem 1.1 will then be a consequence of the next three propositions.

Proposition 5.3. The following properties are equivalent:

(i) $\int_{\mathbb{R}^{d}} p_{t}(x, y)|f(y)| d y<\infty$, for all $t>0$ and $x \in \mathbb{R}^{d}$;

(ii) $\int_{\mathbb{R}^{d}} p_{t_{0}}\left(x_{0}, y\right)|f(y)| d y<\infty$, for some $t_{0}>0$ and some $x_{0} \in \mathbb{R}^{d}$;

(iii) $\int_{\mathbb{R}^{d}}|f(y)| e^{-\sqrt{R\left(1+|y|^{2}\right)}}(1+|y|)^{-\left(\frac{d}{2}+1\right)} d y<\infty$.

Proof. It is clear that (i) $\Rightarrow$ (ii). To see that (ii) $\Rightarrow$ (iii), one uses the left inequality in Lemma 5.1, which inserted into (5.4) easily leads to

$$
p_{t_{0}}\left(x_{0}-y\right) \geq c_{1}\left(t_{0}, x_{0}\right) e^{-\sqrt{R\left(1+|y|^{2}\right)}}(1+|y|)^{-\left(\frac{d}{2}+1\right)}, \quad \text { if } \quad|y|>2 \max \left\{\left|x_{0}\right|, 1\right\} .
$$

Since (ii) also implies that $f$ is locally integrable, the statement in (iii) follows. 
Finally, to see that (iii) $\Rightarrow$ (i), one can again restrict to $|y|>2 \max \{|x|, 1\}$, and then the assertion is a consequence of the right inequality in Lemma 5.1 .

Proposition 5.4. If $f$ satisfies the conditions in Proposition 5.3 , then

$$
u(t, x)=\int_{\mathbb{R}^{d}} p_{t}(x-y) f(y) d y \in C^{\infty}\left((0, \infty) \times \mathbb{R}^{d}\right) .
$$

Proof. We only sketch the proof, as the argument is similar to that in Proposition 4.4. It suffices to consider derivatives with respect to $t$, and one sees from (5.2) that this amounts to studying the decay of the kernels

$$
p_{t, \ell}(x)=\int_{0}^{\infty} e^{-\frac{t^{2}+|x|^{2}}{4 u}} e^{-u R} u^{-\frac{d+3+2 \ell}{2}} d u, \quad \forall \ell \in \mathbb{N} .
$$

Changing variables as in (5.3) and using the asymptotics of the Bessel potentials (now $G_{2 d+2 \ell+1}(x)$ ) one sees that

$$
p_{t, \ell}(x) \approx \frac{t(1+\sqrt{R}(t+|x|))^{\frac{d}{2}+\ell} e^{-\sqrt{R\left(t^{2}+|x|^{2}\right)}}}{(t+|x|)^{d+1+2 \ell}},
$$

which as expected have a better decay than the original kernel. The integrability conditions assumed in $f$ then suffice to give (5.7).

Proposition 5.5. If $f$ satisfies the conditions in Proposition [5.3, then

$$
\lim _{t \rightarrow 0^{+}} e^{-t \sqrt{L}} f(x)=f(x), \quad \text { a.e. } x \in \mathbb{R}^{d} .
$$

Proof. Once Proposition 5.3 has been established, the proof is entirely analogous to that of Proposition 4.5. We leave the details to the reader.

5.3. Proof of Theorem 1.3 for the Poisson equation. Using Lemma 5.2 and arguing as in 4.3 we see that

$$
P_{a}^{*} f(x) \lesssim C_{x} a^{\frac{d}{2}}\left[\mathcal{M}_{2}^{\text {loc }}\left(f e^{-\sqrt{R\left(1+|y|^{2}\right)}}\right)(x)+a\|f\|_{L^{p}(v)}\left\|v^{-\frac{1}{p}} \varphi\right\|_{p^{\prime}}\right],
$$

with $C_{x}=c_{R}(1+|x|)^{\frac{d}{2}} e^{\sqrt{R}|x|}$ and $\varphi(y)=e^{-\sqrt{R\left(1+|y|^{2}\right)}}(1+|y|)^{-\left(\frac{d}{2}+1\right)}$. The right hand term will belong to $L^{p}(u)$ provided we choose

$$
u(x) \leq u_{1}(x):=\frac{1}{C_{x}^{p}(1+|x|)^{d+1}}=c^{\prime} e^{-p \sqrt{R}|x|}(1+|x|)^{-\frac{p d}{2}-d-1} .
$$

Observe that $\left\|u_{1}^{-\frac{\sigma}{p}} \varphi\right\|_{p^{\prime}}<\infty$ for every $\sigma<1$.

For the left hand term in (5.8), the new function $\tilde{f}(y)=f(y) e^{-\sqrt{R\left(1+|y|^{2}\right)}} \in$ $L^{p}(\tilde{v})$ for the weight $\tilde{v}(y)=v(y) e^{p \sqrt{R\left(1+|y|^{2}\right)}}$. This weight satisfies

$$
\left\|\tilde{v}^{-\frac{1}{p}} e^{-\varepsilon|y|}\right\|_{p^{\prime}}=\left\|v^{-\frac{1}{p}} e^{-\varepsilon|y|} e^{-\sqrt{R} \sqrt{1+|y|^{2}}}\right\|_{p^{\prime}}<\infty, \quad \forall \varepsilon>0,
$$

so we can apply Theorem 2.1 (and Remark 2.2) to find, for each $\sigma<1$, a weight $\tilde{U}$ such that

$$
\left\|\mathcal{M}_{2}^{\text {loc }} \tilde{f}\right\|_{L^{p}(\tilde{U})} \lesssim\|\tilde{f}\|_{L^{p}(\tilde{v})}=\|f\|_{L^{p}(v)} \quad \text { and } \quad\left\|\tilde{U}^{-\frac{\sigma}{p}} e^{-\varepsilon|y|}\right\|_{p^{\prime}}<\infty, \quad \forall \varepsilon>0 .
$$

Then, setting

$$
u_{0}(x)=\frac{\tilde{U}(x)}{C_{x}^{p}}=c^{\prime} \tilde{U}(x) e^{-p \sqrt{R}|x|}(1+|x|)^{-d p / 2}
$$


and calling $A f(x)$ the first summand in (5.8), we see that $\|A f\|_{L^{p}\left(u_{0}\right)} \lesssim\|f\|_{L^{p}(v)}$ and

$$
\left\|u_{0}^{-\frac{\sigma}{p}} \varphi\right\|_{p^{\prime}} \leq\left\|\tilde{U}^{-\frac{\sigma}{p}}(1+|x|)^{\frac{d \sigma}{2}} e^{-(1-\sigma) \sqrt{R}|x|}\right\|_{p^{\prime}}<\infty .
$$

Thus, we can finally choose

$$
u(x)=\min \left\{u_{0}(x), u_{1}(x)\right\},
$$

which satisfies the required properties.

\section{The Ornstein-Uhlenbeck operator}

It is well known that the Ornstein-Uhlenbeck operator $\mathcal{O}=-\Delta+2 x \cdot \nabla$ in $\mathbb{R}^{d}$ is closely related to a small perturbation of the Hermite operator

$$
L=-\Delta+|x|^{2}-d, \quad \text { in } \quad \mathbb{R}^{d} .
$$

Indeed, if we set $\tilde{u}(x)=e^{-|x|^{2} / 2} u(x)$, then it is easily seen that $\mathcal{O} u(x)=e^{|x|^{2} / 2}[L \tilde{u}](x)$. Thus,

$$
e^{-t \mathcal{O}} f(x)=e^{\frac{|x|^{2}}{2}} e^{-t L} \tilde{f}(x) \text { and } e^{-t \sqrt{\mathcal{O}}} f(x)=e^{\frac{|x|^{2}}{2}} e^{-t \sqrt{L}} \tilde{f}(x),
$$

so that the convergence properties of $\mathcal{O}$ can be obtained from those of $L$ via the mapping $f \mapsto \tilde{f}$; see e.g. [1, (3.1)]. We sketch below the arguments needed to deal with the operator $L$ in (6.1), and hence obtain Theorems 1.1 and 1.3 for the operator $\mathcal{O}$.

6.1. Proof of Theorem 1.1. Consider first the heat equation associated with $L=-\Delta+|x|^{2}-d$ and datum $\tilde{f}$, whose solution can be written as

$$
e^{-t L} \tilde{f}(x)=e^{t d} e^{-t \mathcal{H}} \tilde{f}(x), \quad \text { with } \quad \mathcal{H}=-\Delta+|x|^{2} .
$$

Clearly (i) and (ii) hold, requiring in $\tilde{f}$ the same conditions as for the operator $\mathcal{H}$ in Table 1. This in turn implies that Theorem 1.1 holds for $u(t, x)=e^{-t \mathcal{O}} f(x)$ provided that

$$
\int_{\mathbb{R}^{d}}|f(y)| e^{-\frac{|y|^{2}}{4}\left(\frac{1}{s}+2\right)} d y<\infty, \quad \forall s \in(0, \operatorname{th}(2 \mathrm{~T}) / 2) .
$$

This is exactly the statement in Table 1 for the operator $\mathcal{O}$.

We now move to the Poisson semigroup associated with $L=-\Delta+|x|^{2}-d$. The subordination formula in this case takes the form

$$
e^{-t \sqrt{L}}=\frac{t}{\sqrt{4 \pi}} \int_{0}^{\infty} e^{-\frac{t^{2}}{4 u}} e^{-u \mathcal{H}} e^{u d} \frac{d u}{u^{3 / 2}} .
$$

Hence, the substitution $s=$ th $u$ (i.e. $u=\frac{1}{2} \ln \frac{1+s}{1-s}$ ) gives the integral expression

$$
p_{t}(x, y)=\frac{t}{(4 \pi)^{\frac{d+1}{2}}} \int_{0}^{1} \frac{e^{-\frac{t^{2}}{2 \ln \frac{1+s}{1-s}}}\left(1-s^{2}\right)^{\frac{d}{2}-1} e^{-\frac{1}{4}\left(\frac{|x-y|^{2}}{s}+s|x+y|^{2}\right)}}{s^{\frac{d}{2}}\left(\frac{1}{2} \ln \frac{1+s}{1-s}\right)^{3 / 2}}\left(\frac{1+s}{1-s}\right)^{\frac{d}{2}} d s .
$$

This is quite similar to the Poisson-Hermite kernel in (4.1), except that the power $(1-s)^{d / 2}$ in the numerator now cancels out with the new term appearing from the factor $e^{u d}$. At this point one can apply the same arguments as in the proof of Lemma 4.1. which produce the estimate

$$
p_{t}(x, y) \approx \frac{e^{-|y|^{2} / 2}}{[\ln (e+|y|)]^{\frac{1}{2}}}, \quad \forall y \in \mathbb{R}^{d}
$$


(modulo multiplicative constants $C=C_{t, x}$ ). We do not carry out the details of (6.4), but these are easily traced looking at the expression for $I_{1}$ in (4.7) (now without the factor $\left.(1-s)^{d / 2}\right)$ and its estimates in (4.11) and (4.10). The loss in the logarithmic power is due to the fact that

$$
\int_{0}^{1 /|y|} \frac{d u}{\left(\ln \frac{2}{u}\right)^{\frac{3}{2}} u} \approx \frac{1}{[\ln (e+|y|)]^{\frac{1}{2}}} \quad \text { and } \quad \int_{0}^{\frac{|y|}{2}} \frac{e^{-c v^{2}} d v}{\left(\ln \frac{|y|}{v}\right)^{\frac{3}{2}} v} \approx \frac{1}{[\ln (e+|y|)]^{\frac{1}{2}}} .
$$

From (6.4) a version of Theorem 1.1 for $L=-\Delta+|x|^{2}-d$ follows exactly as in 4.2. that is, $u(t, x)=e^{-t \sqrt{L}} \tilde{f}(x)$ satisfies (i) + (ii) if and only if

$$
\int_{\mathbb{R}^{d}}|\tilde{f}(y)| e^{-\frac{|y|^{2}}{2}}[\ln (e+|y|)]^{-\frac{1}{2}} d y<\infty .
$$

Using (6.2) this implies that the solution of the Poisson-Ornstein-Uhlenbeck equation $u(t, x)=e^{-t \sqrt{\mathcal{O}}} f(x)$ satisfies the assertions in Theorem 1.1 if and only if

$$
\int_{\mathbb{R}^{d}}|f(y)|[\ln (e+|y|)]^{-\frac{1}{2}} e^{-|y|^{2}} d y<\infty,
$$

as stated in Table 1 .

6.2. Proof of Theorem 1.3. We first consider the maximal heat operators associated with $L=-\Delta+|x|^{2}-d$ and $\mathcal{O}$. Observe that they are related by

$$
h_{a}^{*, \mathcal{O}} f(x)=e^{\frac{|x|^{2}}{2}} h_{a}^{*, L} \tilde{f}(x) \quad \text { and } \quad h_{a}^{*, L} \tilde{f}(x) \leq e^{a d} h_{a}^{*, \mathcal{H}} \tilde{f}(x) .
$$

Clearly, $h_{a}^{*, L}$ inherits the same boundedness properties of $h_{a}^{*, \mathcal{H}}$, and we shall use these to prove Theorem 1.3 for $h_{a}^{*, \mathcal{O}}$.

Let $v \in D_{p}^{\text {heat }}(\mathcal{O})$, which according to Corollary 1.2 means that

$$
\left\|v^{-\frac{1}{p}} e^{-\frac{|y|^{2}}{4}\left(\frac{1}{s}+2\right)}\right\|_{p^{\prime}}<\infty, \quad \forall s<\operatorname{th}(2 \mathrm{~T}) / 2 .
$$

Then, the weight $V(y)=v(y) e^{\frac{p|y|^{2}}{2}}$ belongs to $D_{p}^{\text {heat }}(\mathcal{H})$. By Theorem 1.3 applied to $\mathcal{H}$, there is a $\sigma_{0}=\sigma_{0}\left(a, \mathrm{~T}^{*}\right) \in(0,1)$ such that, for any $\sigma<\sigma_{0}$, there exists some weight $U$ such that $h_{a}^{*, \mathcal{H}}: L^{p}(V) \rightarrow L^{p}(U)$ and $U^{\sigma} \in D_{p}^{\text {heat }}(\mathcal{H})$. Now consider the weight $u(x)=e^{-\frac{p|x|^{2}}{2 \sigma}} U(x)$. Clearly $u^{\sigma} \in D_{p}^{\text {heat }}(\mathcal{O})$, while

$$
\begin{aligned}
\left\|h_{a}^{*, \mathcal{O}} f\right\|_{L^{p}(u)} & =\left\|e^{\frac{|x|^{2}}{2}} h_{a}^{*, L} \tilde{f}(x) e^{-\frac{|x|^{2}}{2 \sigma}}\right\|_{L^{p}(U)} \\
& \leq e^{a d}\left\|h_{a}^{*, \mathcal{H}} \tilde{f}\right\|_{L^{p}(U)} \lesssim\|\tilde{f}\|_{L^{p}(V)}=\|f\|_{L^{p}(v)} .
\end{aligned}
$$

We now pass to the maximal Poisson operators. As before we have

$$
P_{a}^{*, \mathcal{O}} f(x)=e^{\frac{|x|^{2}}{2}} P_{a}^{*, L} \tilde{f}(x),
$$

so it suffices to work with the expression $P_{a}^{*, L} \tilde{f}$. To do so we need an estimate of the Poisson kernel $p_{t}^{L}(x, y)$ of $L=-\Delta+|x|^{2}-d$, of the form

$$
p_{t}^{L}(x, y) \leq C_{x}\left[p_{t}^{\Delta}(x-y) e^{-\frac{|y|^{2}}{2}} \chi_{\{|y| \leq \gamma \max \{|x|, 1\}\}}+t \varphi(y)\right]
$$


with $\varphi(y)=[\ln (e+|y|)]^{-\frac{1}{2}} e^{-|y|^{2} / 2}$. This corresponds to formula (4.12) for the Hermite-Poisson kernel and can be obtained with obvious modifications in the proof; namely, the integral $I_{1}$ must now be estimated by the right hand side of (6.4).

At this point one proceeds as in $\$ 4.3$, with this new expression of $\varphi(y)$, to obtain that, for every weight $V \in D_{p}^{\text {Pois }}(L)$, that is,

$$
\left\|V^{-\frac{1}{p}} \varphi\right\|_{L^{p^{\prime}\left(\mathbb{R}^{d}\right)}}<\infty
$$

and every $\sigma<1$, there exists a weight $U$ such that $P_{a}^{*, L}: L^{p}(V) \rightarrow L^{p}(U)$ and $U^{\sigma} \in D_{p}^{\text {Pois }}(L)$. Finally, let $v \in D_{p}^{\text {Pois }}(\mathcal{O})$. By $(\underline{6.2})$, the weight $V(y)=v(y) e^{\frac{p|y|^{2}}{2}}$ belongs to $D_{p}^{\text {Pois }}(L)$, so we can apply the previous result to obtain a weight $U$. Next, consider $u(x)=e^{-\frac{p|x|^{2}}{2 \sigma}} U(x)$, which satisfies $u^{\sigma} \in D_{p}^{\text {Pois }}(\mathcal{O})$, and

$$
\begin{aligned}
\left\|P_{a}^{*, \mathcal{O}} f\right\|_{L^{p}(u)} & =\left\|e^{\frac{|x|^{2}}{2}} P_{a}^{*, L} \tilde{f}(x) e^{-\frac{|x|^{2}}{2 \sigma}}\right\|_{L^{p}(U)} \\
& \leq\left\|P_{a}^{*, L} \tilde{f}\right\|_{L^{p}(U)} \lesssim\|\tilde{f}\|_{L^{p}(V)}=\|f\|_{L^{p}(v)} .
\end{aligned}
$$

This establishes Theorem 1.3 for the maximal Poisson-Ornstein-Uhlenbeck operator.

\section{FURTHER COMMENTS}

7.1. An example. Consider the classical heat equation $u_{t}=\Delta u$ in $(0, \mathrm{~T}) \times \mathbb{R}^{d}$, with initial datum $f(y)=e^{|y|^{2} /(4 \mathrm{~T})}$. An explicit solution is given by

$$
h_{t} * f(x)=\left(1-\frac{t}{\mathrm{~T}}\right)^{-d / 2} e^{\frac{|x|^{2}}{4(\mathrm{~T}-t)}}, \quad t \in(0, \mathrm{~T}), x \in \mathbb{R}^{d} .
$$

Clearly, $f \in L^{p}(v)$ with $v(y)=(1+|y|)^{-d-1} e^{-p|y|^{2} /(4 \mathrm{~T})}$, which belongs to the class $D_{p}^{\text {heat }}$ associated with $\Delta$ in $(0, \mathrm{~T}) \times \mathbb{R}^{d}$ as described in (1.6). However, notice that the function $h_{a} * f(x)$ has a much larger exponential growth than $f$ (if $a$ is close to $\mathrm{T})$. Hence if one expects $h_{a} * f$ to belong to $L^{p}(u)$, the weight $u$ must satisfy stronger conditions than $v$.

For example, one could choose $u(x)=(1+|x|)^{-d-1} e^{-p|x|^{2} /[4(T-a)]}$, but then $u^{\sigma}$ will only belong to $D_{p}^{\text {heat }}$ if $\sigma \leq(T-a) / T$. This shows the necessity of a restriction in $\sigma$ (depending on $a$ and $\mathrm{T}$ ) for the $L^{p}(v) \rightarrow L^{p}(u)$ boundedness of maximal heat operators, as stated in Theorem 1.3 .

7.2. Non-tangential convergence. For every $\alpha>0$, the statement of Theorem 1.1 can be improved to

$$
\lim _{\substack{(t, x) \rightarrow\left(0, x_{0}\right) \\\left|x-x_{0}\right|^{2}<\alpha t}} e^{-t L} f(x)=f\left(x_{0}\right) \quad \text { and } \quad \lim _{\substack{(t, x) \rightarrow\left(0, x_{0}\right) \\\left|x-x_{0}\right|<\alpha t}} e^{-t \sqrt{L}} f(x)=f\left(x_{0}\right), \quad \text { a.e. } x_{0} \in \mathbb{R}^{d} .
$$

Indeed, non-tangential convergence is known to hold for $L^{1}$-functions [12, and hence, going back to the proofs of Propositions 3.5 and 4.5, such result could be applied to the "local" part of $f, f_{1}=f \chi_{\{|y| \leq 2 n\}}$ in formulas (3.11) and (4.18). The remainder terms $f_{2}=f \chi_{\{|y|>2 n\}}$ would not be a problem, since their estimates in (3.10) and (4.17) are uniform in $x$. 
Similarly, Theorem 1.3 will continue to hold if we replace $h_{a}^{*}$ and $P_{a}^{*}$ by their parabolic and non-tangential analogues, respectively,

$$
h_{a}^{*, \alpha} f\left(x_{0}\right)=\sup _{\substack{0<t<a \\\left|x-x_{0}\right|^{2}<\alpha t}}\left|e^{-t L} f(x)\right| \quad \text { and } \quad P_{a}^{*, \alpha} f\left(x_{0}\right)=\sup _{\substack{0<t<a \\\left|x-x_{0}\right|<\alpha t}}\left|e^{-t \sqrt{L}} f(x)\right|,
$$

and every fixed $\alpha>0$. The modifications are again standard and left to the reader.

7.3. Alternative approach to the Hermite heat operator. Propositions 3.3 and 3.5 can be proved in a slightly faster way using an alternative expression for the Mehler kernel, which relates it directly to the classical heat kernel. Setting $s=\operatorname{th}(2 t) / 2$ one has

$$
\begin{aligned}
h_{t}(x, y) & =\left(1-4 s^{2}\right)^{\frac{d}{4}} e^{-s|x|^{2}} \frac{e^{-\left|\sqrt{1-4 s^{2}} x-y\right|^{2} /(4 s)}}{(4 \pi s)^{\frac{d}{2}}} \\
& =\left(1-4 s^{2}\right)^{\frac{d}{4}} e^{-s|x|^{2}} W_{s}\left(\sqrt{1-4 s^{2}} x-y\right)
\end{aligned}
$$

(see [9] and also [2, 14]). For instance, Proposition 3.5] will then follow from the pointwise non-tangential convergence of the classical heat equation. For the Poisson equation, however, this approach does not seem to give easier formulas.

7.4. Global maximal functions. One may ask what would be the role of the "global" maximal functions $h^{*} f(x)$ and $P^{*} f(x)$ defined in (1.1). These are related to the characterization of the functions $f$ whose heat or Poisson integrals, in addition to (i) and (ii) (with $\mathrm{T}=\infty$ ), also satisfy

$$
\exists \lim _{t \rightarrow \infty} u(t, x), \quad \text { a.e. } x \in \mathbb{R}^{d} .
$$

This is a different problem, which for the heat equation associated with the OrnsteinUhlenbeck operator was investigated in [8]. In the remaining cases, it seems to be open.

7.5. Extensions to fractional Laplacian operators. The results obtained in this paper for the Poisson equation $(\mathrm{P})$ actually hold in a slightly more general setting. Given $\sigma>0$ consider the pde

$$
u_{t t}+\frac{1-2 \sigma}{t} u_{t}=L u \quad \text { in } \mathbb{R}_{+}^{d+1}, \quad \text { with } u(0)=f,
$$

which for $\sigma=1 / 2$ coincides with (P). A formal solution is given by

$$
u(t, x)=\frac{t^{2 \sigma}}{4^{\sigma} \Gamma(\sigma)} \int_{0}^{\infty} e^{-\frac{t^{2}}{4 u}} e^{-u L} f(x) \frac{d u}{u^{1+\sigma}}, \quad t>0 ;
$$

see [18, Thm. 1.1]. This example is relevant in the theory of fractional operators since, under sufficiently good conditions on $f$, one can recover $L^{\sigma}$ by

$$
\lim _{t \rightarrow 0} t^{1-2 \sigma} u_{t}(t, x)=c_{\sigma} L^{\sigma} f(x) .
$$

Notice that (7.2) is only a slight generalization of (1.5), so estimates for the corresponding kernel $p_{t}^{(\sigma)}(x, y)$ can be obtained exactly as in Lemma 4.1, with the outcome

$$
\varphi^{(\sigma)}(y)=(1+|y|)^{-d / 2}[\ln (e+|y|)]^{-(1+\sigma)} e^{-|y|^{2} / 2} .
$$

We leave a more detailed study of these operators for a subsequent work. 


\section{ACKNOWLEDGEMENTS}

This research was carried out during a visit of the first and third authors to the Instituto de Matemática Aplicada del Litoral (UNL-CONICET), Santa Fe, Argentina, in July 2013. The authors wish to thank people in this institution for their very kind support and hospitality. The authors also thank an anonymous referee for a careful reading and many suggestions (including a correct and more elegant proof of Lemma 4.1), which certainly have improved the preliminary version of this paper.

\section{REFERENCES}

[1] Ibraheem Abu-Falahah, Pablo Raúl Stinga, and José L. Torrea, A note on the almost everywhere convergence to initial data for some evolution equations, Potential Anal. 40 (2014), no. 2, 195-202, DOI 10.1007/s11118-013-9351-z. MR3152161

[2] V. Aldaya, F. Cossío, J. Guerrero, and F. F. López-Ruiz, The quantum Arnold transformation, J. Phys. A 44 (2011), no. 6, 065302, 19, DOI 10.1088/1751-8113/44/6/065302. MR2763443(2012d:81140)

[3] N. Aronszajn and K. T. Smith, Theory of Bessel potentials. I (English, with French summary), Ann. Inst. Fourier (Grenoble) 11 (1961), 385-475. MR0143935 (26 \#1485)

[4] Colin Bennett and Robert Sharpley, Interpolation of operators, Pure and Applied Mathematics, vol. 129, Academic Press, Inc., Boston, MA, 1988. MR928802 (89e:46001)

[5] L. Carleson and P. Jones, Weighted norm inequalities and a theorem of Koosis, Mittag-Leffler Inst., report n. 2, 1981.

[6] José García-Cuerva and José L. Rubio de Francia, Weighted norm inequalities and related topics, North-Holland Mathematics Studies, vol. 116, Notas de Matemática [Mathematical Notes], 104, North-Holland Publishing Co., Amsterdam, 1985. MR807149 (87d:42023)

[7] Silvia I. Hartzstein, José L. Torrea, and Beatriz E. Viviani, A note on the convergence to initial data of heat and Poisson equations, Proc. Amer. Math. Soc. 141 (2013), no. 4, 13231333, DOI 10.1090/S0002-9939-2012-11441-8. MR3008879

[8] E. Harboure, J. L. Torrea, and B. Viviani, On the search for weighted inequalities for operators related to the Ornstein-Uhlenbeck semigroup, Math. Ann. 318 (2000), no. 2, 341-353, DOI 10.1007/PL00004424. MR 1795566 (2002b:42029)

[9] Bang-He Li, Explicit relation between the solutions of the heat and the Hermite heat equation, Z. Angew. Math. Phys. 58 (2007), no. 6, 959-968, DOI 10.1007/s00033-007-7044-4. MR2363895(2009a:35102)

[10] L. Liu and P. Sjögren, A characterization of the Gaussian Lipschitz space and sharp estimates for the Ornstein-Uhlenbeck Poisson kernel, to appear in Rev. Matem. Iberoamericana.

[11] E. M. Nikishin, A resonance theorem and series in eigenfunctions of the Laplace operator (Russian), Izv. Akad. Nauk SSSR Ser. Mat. 36 (1972), 795-813; English transl., Math. USSR Izv. 6 (4) (1972), 788-806. MR0343091 (49 \#7835)

[12] Ebner Pineda and Wilfredo Urbina R., Non tangential convergence for the OrnsteinUhlenbeck semigroup (English, with English and Spanish summaries), Divulg. Mat. 16 (2008), no. 1, 107-124. MR2587011 (2010m:60121)

[13] José L. Rubio de Francia, Weighted norm inequalities and vector valued inequalities, Harmonic analysis (Minneapolis, Minn., 1981), Lecture Notes in Math., vol. 908, Springer, BerlinNew York, 1982, pp. 86-101. MR654181 (83h:42025)

[14] Peter Sjögren and J. L. Torrea, On the boundary convergence of solutions to the HermiteSchrödinger equation, Colloq. Math. 118 (2010), no. 1, 161-174, DOI 10.4064/cm118-1-8. MR.2600523 (2011b:42078)

[15] Elias M. Stein, Topics in harmonic analysis related to the Littlewood-Paley theory, Annals of Mathematics Studies, No. 63, Princeton University Press, Princeton, N.J.; University of Tokyo Press, Tokyo, 1970. MR0252961 (40 \#6176)

[16] Elias M. Stein, Singular integrals and differentiability properties of functions, Princeton Mathematical Series, No. 30, Princeton University Press, Princeton, N.J., 1970. MR0290095 (44 \#7280) 
[17] Elias M. Stein, Harmonic analysis: real-variable methods, orthogonality, and oscillatory integrals, Princeton Mathematical Series, vol. 43, Princeton University Press, Princeton, NJ, 1993. With the assistance of Timothy S. Murphy; Monographs in Harmonic Analysis, III. MR $1232192(95 \mathrm{c}: 42002)$

[18] Pablo Raúl Stinga and José Luis Torrea, Extension problem and Harnack's inequality for some fractional operators, Comm. Partial Differential Equations 35 (2010), no. 11, 20922122, DOI 10.1080/03605301003735680. MR2754080 (2012c:35456)

[19] Krzysztof Stempak and José Luis Torrea, Poisson integrals and Riesz transforms for Hermite function expansions with weights, J. Funct. Anal. 202 (2003), no. 2, 443-472, DOI 10.1016/S0022-1236(03)00083-1. MR1990533(2004d:42024)

[20] A. Tychonoff, Théorèmes d'unicité pour l'équation de la chaleur, Math. Sb. 42 (2) (1935), 199-216.

[21] D. V. Widder, The heat equation, Pure and Applied Mathematics, Vol. 67, Academic Press [Harcourt Brace Jovanovich, Publishers], New York-London, 1975. Pure and Applied Mathematics, Vol. 67. MR0466967 (57 \#6840)

[22] Calvin H. Wilcox, Positive temperatures with prescribed initial heat distributions, Amer. Math. Monthly 87 (1980), no. 3, 183-186, DOI 10.2307/2321603. MR562921 (81i:35077)

Departamento de Matemáticas, Universidad de Murcia, 30100 Murcia, Spain

E-mail address: gustavo.garrigos@um.es

IMAL (UNL-CONICET) y FIQ (Universidad Nacional del Litoral), Güemes 3450, 3000 Santa Fe, Argentina

E-mail address: shartzstein@santafe-conicet.gov.ar

Departamento de Matemáticas, Universidad de Murcia, 30100 Murcia, Spain

E-mail address: tmsignes@um.es

Departamento de Matemáticas, Universidad Autónoma de Madrid, iCMAT-CisCUAM-UCM-UC3M, 28049, MADRID, SPAIN

E-mail address: joseluis.torrea@uam.es

IMAL (UNL-CONICET) y FIQ (Universidad Nacional del Litoral), CCT CONICET Santa Fe Colectora Ruta Nac. N168, Paraje El Pozo, 3000 Santa Fe, Argentina

E-mail address: viviani@santafe-conicet.gov.ar 